\title{
Comparing the $\pi$-primary submodules of the dual Selmer groups
}

\author{
Meng Fai Lim*
}

\begin{abstract}
In this paper, we compare the structure of Selmer groups of certain classes of Galois representations over an admissible $p$-adic Lie extension. Namely, we show that the $\pi$-primary submodules of the Pontryagin dual of the Selmer groups of two Galois representations have the same elementary representations when two Galois representations in question are either Tate dual to each other or are congruent to each other. In the first situation, our result gives a partial answer to the question of Greenberg on whether the Pontryagin dual of the Selmer groups of two Galois representations that are Tate dual to each other are pseudo-isomorphic (up to a twist of the Iwasawa algebra). In the second situation, our result will be applied to study the variation of the $\pi$-primary submodules of the dual Selmer groups of certain specialization of a big Galois representation. One of the important ingredient in our proofs is an asymptotic formula for $\pi$-primary modules over a noncommutative Iwasawa algebra which can be viewed as a generalization of a weak analog of the classical Iwasawa asymptotic formula.
\end{abstract}

Keywords and Phrases: Selmer groups, $\pi$-primary submodules, elementary representations, admissible $p$-adic Lie extensions, asymptotic formula.

Mathematics Subject Classification 2010: 11R23, 11R34, 11F80.

\section{Introduction}

Throughout the paper, $p$ will always denote a rational prime. Let $\mathcal{O}$ be the ring of integers of a fixed finite extension $K$ of $\mathbb{Q}_{p}$, and let $\pi$ be a local parameter of $\mathcal{O}$. Let $F$ be a number field and $F_{\infty}$ an admissible $p$-adic Lie extension of $F$ with Galois group $G$. For a Galois representation defined over a number field $F$ with coefficients in $\mathcal{O}$, one can attach a Selmer group to these data, and the resulting Selmer group has a natural module structure over $\mathcal{O} \llbracket G \rrbracket$ which is the Iwasawa algebra of the Galois group of the given admissible $p$-adic Lie extension. For a module over such an Iwasawa algebra, Howson [Ho1] and Venjakob [V1] independently developed the notion of a generalized $\mu$-invariant which extends the classical notion of the $\mu$-invariant. Building on

${ }^{*}$ School of Mathematics and Statistics \& Hubei Key Laboratory of Mathematical Sciences, Central China Normal University, Wuhan, 430079, P.R.China. E-mail: limmf@mail.ccnu.edu.cn 
this notion of the $\mu$-invariant, they have established a structural description of the $\pi$-primary submodules of modules defined over such an Iwasawa algebra (see [Ho2, V1]). To be precise, they are able to show that the $\pi$-primary submodules can be expressed uniquely in term of a product of factors of the form $\mathcal{O} \llbracket G \rrbracket / \pi^{\alpha}$ (up to pseudo-isomorphism). Following the classical situation, we call this the elementary representation of the $\pi$-primary submodule. In the midst of establishing their structural theorem, they also show that the $\mu$-invariant can be expressed as a sum of the $\alpha$ 's appearing in the elementary representation. The aim of this article is to compare the $\pi$-primary submodules of the Pontryagin dual of the Selmer groups of two Galois representations in the two situations as mentioned in the abstract. We briefly describe these situations in the next two paragraphs.

The main conjecture of Iwasawa theory is a conjecture on the relation between a Selmer group and a conjectural $p$-adic $L$-function (see $\left[\mathrm{CF}^{+}, \mathrm{FK}, \mathrm{Gr} 1\right]$ ). This $p$-adic $L$-function is expected to satisfy a conjectural functional equation in a certain sense. In view of the main conjecture and this conjectural functional equation, one would expect to have certain algebraic relationship between the Selmer group attached to a Galois representation and the Selmer group attached to the Tate twist of the dual of the Galois representation which can be thought as an algebraic manifestation of the functional equation. In particular, an immediate consequence of this algebraic functional equation is that the Selmer group attached to a Galois representation and the Selmer group attached to the Tate twist of the dual representation have the same generalized Iwasawa $\mu$-invariants. In the case of a cyclotomic $\mathbb{Z}_{p}$-extension, this study on the $\mu$-invariants has been undertaken in Gr1, Mat. (Actually, in Gr1], Greenberg also established the full "algebraic" functional equation of the Selmer groups, which we will not treat in this article. Readers interested in this subject may refer to [BZ, Hs, JP, LLTT, Z1, Z2.) For a noncommutative $p$-adic Lie extension, this equality has been verified for Selmer groups of an abelian variety without complex multiplication (see [Bh] $)$. In a previous unpublished note $[\mathrm{Lim}]$, the author has also verified the equality of the $\mu$-invariants. In this paper, we will show an even stronger relation, namely, the $\pi$-primary submodules of the dual Selmer groups in question have the same elementary representations. In particular, our result will imply the equality of the generalized Iwasawa $\mu$-invariants. We like to emphasize that although this latter result is motivated by the main conjecture of Iwasawa and the functional equation of the conjectural $p$ adic $L$-functions, we do not assume these conjectures in all our argument. We also mention that our result answers the $\pi$-primary part of the question of Greenberg on whether the Pontryagin dual of the Selmer groups of two Galois representations are pseudo-isomorphic (up to a twist of the Iwasawa algebra).

The second situation is concerned with comparing the Selmer groups of two congruent Galois 
representations, or in general, a family of Galois representations with suitable congruence relations. Such studies were carried out over the cyclotomic $\mathbb{Z}_{p}$-extension in [EPW, Gr2, GV, Ha, We and over noncommutative $p$-adic Lie extensions in [Ch, $[\mathrm{SS}$. One of the motivations behind these studies lies in the philosophy that the "Iwasawa main conjecture" should be preserved by congruences. We should mention that in the cyclotomic case, this philosophy is rather well understood (see [EPW, GV, Oc]), although the general noncommutative situation still seems much of a mystery (for instance, see $[\mathrm{B}, \mathrm{CS}]$ ). An important observation made in most of the above cited works is that if the Iwasawa $\mu$-invariant of one of the Selmer groups vanishes, so does the other. It is then natural to consider the situation when the said Iwasawa $\mu$-invariants are nonzero and ask if one can relate the Iwasawa $\mu$-invariants. To the best of the author's knowledge, such studies have only been considered in [B, BS]. As observed in these works and as we will see in this paper, to be able to even compare the Iwasawa $\mu$-invariants meaningfully, we require the congruence of the Galois representations to be high enough. In this paper, we will show the stronger assertion, namely, that the $\pi$-primary submodules of the Selmer groups of congruent Galois representations have the same elementary representations.

We now describe briefly the idea behind the comparison of the $\pi$-primary submodules of the dual Selmer groups of two Galois representations defined over some number field $F$. Let $A$ and $B$ be quotient modules of the two Galois representations. Here the quotient module is obtained from the Galois representation by taking the quotient of the representation by a Galois-invariant lattice. Then one can attach dual Selmer groups to these quotient modules (see Section 3) which we denote to be $X\left(A / F_{\infty}\right)$ and $X\left(B / F_{\infty}\right)$, where $F_{\infty}$ is an admissible $p$-adic Lie extension whose Galois group is a uniform pro- $p$ group of dimension $r$. It follows from the theory developed in Subsection 2.4 that to compare $\pi$-primary submodules of the dual Selmer groups, we need to show the equality

$$
\mu_{\mathcal{O} \llbracket G \rrbracket}\left(X\left(A / F_{\infty}\right) / \pi^{n}\right)=\mu_{\mathcal{O} \llbracket G \rrbracket}\left(X\left(B / F_{\infty}\right) / \pi^{n}\right)
$$

for enough $n$ (see Propositions 2.4.6 and 2.4.7 for details). To prove the above equality, we proceed by establishing the following estimate

$$
\left|\left(\mu_{\mathcal{O} \llbracket G \rrbracket}\left(X\left(A / F_{\infty}\right) / \pi^{n}\right)-\mu_{\mathcal{O} \llbracket G \rrbracket}\left(X\left(B / F_{\infty}\right) / \pi^{n}\right)\right) p^{r m}\right|=O\left(p^{(r-1) m}\right)
$$

for each fixed $n$. If we denote $F_{m}$ to be the intermediate extension of $F$ in $F_{\infty}$ corresponding to fixed field of the $(m+1)$-term of the lower $p$-series of $\operatorname{Gal}\left(F_{\infty} / F\right)$, we then have the following inequality 


$$
\begin{aligned}
& \left|\left(\mu_{\mathcal{O} \llbracket G \rrbracket}\left(X\left(A / F_{\infty}\right) / \pi^{n}\right)-\mu_{\mathcal{O} \llbracket G \rrbracket}\left(X\left(B / F_{\infty}\right) / \pi^{n}\right)\right) p^{r m}\right| \leq \\
& \left|\mu_{\mathcal{O} \llbracket G \rrbracket}\left(X\left(A / F_{\infty}\right) / \pi^{n}\right) p^{r m}-\operatorname{ord}_{q}\left(S\left(A / F_{\infty}\right)\left[\pi^{n}\right]^{G_{m}}\right)\right| \\
& +\left|\mu_{\mathcal{O} \llbracket G \rrbracket}\left(X\left(B / F_{\infty}\right) / \pi^{n}\right) p^{r m}-\operatorname{ord}_{q}\left(S\left(B / F_{\infty}\right)\left[\pi^{n}\right]^{G_{m}}\right)\right| \\
& +\operatorname{ord}_{q}\left(S\left(A\left[\pi^{n}\right] / F_{m}\right)\right)-\operatorname{ord}_{q}\left(S\left(A / F_{\infty}\right)\left[\pi^{n}\right]^{G_{m}}\right) \mid \\
& +\operatorname{ord}_{q}\left(S\left(B\left[\pi^{n}\right] / F_{m}\right)\right)-\operatorname{ord}_{q}\left(S\left(B / F_{\infty}\right)\left[\pi^{n}\right]^{G_{m}}\right) \mid \\
& +\left|\operatorname{ord}_{q}\left(S\left(A\left[\pi^{n}\right] / F_{m}\right)\right)-\operatorname{ord}_{q}\left(S\left(B\left[\pi^{n}\right] / F_{m}\right)\right)\right| \text {. }
\end{aligned}
$$

Therefore, we are reduced to showing that the five quantities on the right are $O\left(p^{(r-1) m}\right)$ (for a fixed $n$ ). The estimates for the first and second quantities are where we require the asymptotic formulas for $\pi$-primary modules. The estimates for the third and fourth quantities follow from a descent argument. The estimate of the final quantity is where we make use of the facts that $A$ and $B$ are either Tate dual to each other or congruent to each other for a high enough power. In the case that $A$ and $B$ are Tate dual to each other, our argument follows the approach of Greenberg in [Gr1]. We like to highlight that in the situation of a cyclotomic $\mathbb{Z}_{p}$-extension, the error quantities have bounded order in the intermediate sub-extensions of the cyclotomic $\mathbb{Z}_{p}$-extension. However, since we may have infinite decomposition of primes over a $p$-adic Lie extension of dimension $r>1$, the error quantities may not be bounded, and therefore, we will need a slightly more careful argument.

We now give a brief description of the layout of the paper. In Section 2, we recall certain algebraic notion which will be used subsequently in the paper. It is also here where we develop a method to compare the $\pi$-primary submodules of two $\mathcal{O} \llbracket G \rrbracket$-modules. In the final subsection, we will prove an asymptotic formula for $\pi$-primary modules over an Iwasawa algebra of a (possibly noncommutative) uniform pro- $p$ group. As seen in the previous paragraph, this asymptotic formula is crucial in establishing our main results.

In Section 3, we introduce the Selmer groups which are the main object of study in this paper. These Selmer groups are defined for a set of data which arises from an ordinary Galois representation. Actually, to be precise, the Selmer group that we consider is called the strict Selmer group in Greenberg's terminology [Gr1]. We will also introduce another variant of the Selmer group (called the Greenberg Selmer group) and an appropriate Selmer complex closely related to the strict Selmer group we are working with. In Section 4, we will prove our main theorems for the strict Selmer groups. But it will follow from the discussion of Section 3 that all our main results also hold for the Greenberg Selmer group and the Selmer complex. In Section 5. we will apply our main results to study the variation of the $\pi$-primary submodules of the dual Selmer groups of the specializations of a big Galois representation. When the $p$-adic Lie 
extension is of dimension 2, we will also apply our main results to study the variation of the $\mathfrak{M}_{H}(G)$-property of the dual Selmer groups.

Acknowledgments. A significant portion of this work was written up when the author was a Postdoctoral fellow at the GANITA Lab at the University of Toronto. He would like to acknowledge the hospitality and conducive working conditions provided by the GANITA Lab and the University of Toronto while this work was in progress. The author would also like to thank Otmar Venjakob for answering his question on the asymptotic formulas. The author is also supported by the National Natural Science Foundation of China under the Research Fund for International Young Scientists (Grant No: 11550110172).

\section{Algebraic Preliminaries}

In this section, we recall some algebraic preliminaries that will be required in the later part of the paper. Namely, we gather various notation and definitions which will be required for the discussion of the paper. In Subsection 2.4, we develop various criterions which allow us to compare the structure of the $\pi$-primary submodules of two modules. In the final subsection of this section, we will prove a asymptotic formula over an Iwasawa algebra of a uniform pro- $p$ group which is a generalization of a weak analog to the classical Iwasawa asymptotic formula over an Iwasawa algebra of $\Gamma \cong \mathbb{Z}_{p}[\mathrm{Iw}]$.

\subsection{Compact $p$-adic Lie group}

Fix a prime $p$. In this subsection, we recall some facts about compact $p$-adic Lie groups. The standard references for the material presented here are [DS+, Laz].

For a finitely generated pro- $p$ group $G$, we write $G^{p^{i}}=\left\langle g^{p^{i}} \mid g \in G\right\rangle$, that is, the group generated by the $p^{i}$ th-powers of elements in $G$. The pro- $p$ group $G$ is said to be powerful if $G / \overline{G^{p}}$ is abelian for odd $p$, or if $G / \overline{G^{4}}$ is abelian for $p=2$. We define the lower $p$-series by $P_{1}(G)=G$, and

$$
P_{i+1}(G)=\overline{P_{i}(G)^{p}\left[P_{i}(G), G\right]}, \text { for } i \geq 1 \text {. }
$$

It follows from DS+, Thm. 3.6] that if $G$ is a powerful pro- $p$ group, then $G^{p^{i}}=P_{i+1}(G)$ and the $p$-power map

$$
P_{i}(G) / P_{i+1}(G) \stackrel{\cdot p}{\longrightarrow} P_{i+1}(G) / P_{i+2}(G)
$$

is surjective for each $i \geq 1$. If the $p$-power maps are isomorphisms for all $i \geq 1$, we say that $G$ is uniformly powerful (abrev. uniform). Note that in this case, we have an equality $\left|G: P_{2}(G)\right|=$ 
$\left|P_{i}(G): P_{i+1}(G)\right|$ for every $i \geq 1$. In fact, it is not difficult to see that $\left|G: P_{i+1}(G)\right|=p^{i r}$, where $r=\operatorname{dim} G$.

We now recall the following characterization of compact $p$-adic Lie groups due to Lazard [Laz] (see also DS+, Cor. 8.34]): a topological group $G$ is a compact $p$-adic Lie group if and only if $G$ contains a open normal uniform pro- $p$ subgroup. Furthermore, if $G$ is a compact $p$-adic Lie group without $p$-torsion, it follows from [Ser, Corollaire 1] (see also [Laz, Chap. V Sect. 2.2)]) that $G$ has finite $p$-cohomological dimension.

\subsection{Torsion modules and pseudo-null modules}

As before, $p$ will denote a fixed prime. Let $\mathcal{O}$ be the ring of integers of a finite extension of $\mathbb{Q}_{p}$. For a compact $p$-adic Lie group $G$, the completed group algebra of $G$ over $\mathcal{O}$ is given by

$$
\mathcal{O} \llbracket G \rrbracket=\lim _{U} \mathcal{O}[G / U]
$$

where $U$ runs over the open normal subgroups of $G$ and the inverse limit is taken with respect to the canonical projection maps.

When $G$ is pro- $p$ and has no $p$-torsion, it is well known that $\mathcal{O} \llbracket G \rrbracket$ is an Auslander regular ring (cf. [V1, Theorem 3.26]; for the definition of Auslander regular rings, see [V1, Definition 3.3]). Furthermore, the ring $\mathcal{O} \llbracket G \rrbracket$ has no zero divisors (cf. [Neu $)$, and therefore, admits a skew field $Q(G)$ which is flat over $\mathcal{O} \llbracket G \rrbracket$ (see [GW, Chapters 6 and 10] or [Lam, Chapter 4, $\S 9$ and $\S 10]$ ). If $M$ is a finitely generated $\mathcal{O} \llbracket G \rrbracket$-module, we define the $\mathcal{O} \llbracket G \rrbracket$-rank of $M$ to be

$$
\operatorname{rank}_{\mathcal{O} \llbracket G \rrbracket} M=\operatorname{dim}_{Q(G)} Q(G) \otimes_{\mathcal{O} \llbracket G \rrbracket} M .
$$

We will say that an $\mathcal{O} \llbracket G \rrbracket$-module $M$ is torsion if $\operatorname{rank}_{\mathcal{O} \llbracket G \rrbracket} M=0$. As we will also need to work with various equivalent formulations of a torsion $\mathcal{O} \llbracket G \rrbracket$-module, we state the following.

Lemma 2.2.1. Let $\Lambda$ be a Auslander regular ring with no zero divisors. Let $M$ be a finitely generated $\Lambda$-module. Then the following are equivalent.

(a) The canonical map $\phi: M \longrightarrow \operatorname{Hom}_{\Lambda}\left(\operatorname{Hom}_{\Lambda}(M, \Lambda), \Lambda\right)$ is zero.

(b) $Q(\Lambda) \otimes_{\Lambda} M=0$, where $Q(\Lambda)$ is the skew field of $\Lambda$.

(c) $\operatorname{Hom}_{\Lambda}(M, \Lambda)=0$.

Proof. The equivalence of (a) and (c) follows from [V1, Remark 3.7]. Suppose that $Q(\Lambda) \otimes_{\Lambda} M=$ 0 . Let $f \in \operatorname{Hom}_{\Lambda}(M, \Lambda)$ and $x \in M$. Then since $Q(\Lambda) \otimes_{\Lambda} M=0$, there exists $\lambda \in \Lambda \backslash\{0\}$ such that $\lambda x=0$. This in turn implies that $\lambda f(x)=f(\lambda x)=0$. Since $\Lambda$ has no zero divisor, we have $f(x)=0$. This shows that $\operatorname{Hom}_{\Lambda}(M, \Lambda)=0$ and the implication (b) $\Rightarrow(\mathrm{c})$. Conversely, suppose 
that $\operatorname{Hom}_{\Lambda}(M, \Lambda)=0$. Write $M^{++}=\operatorname{Hom}_{\Lambda}\left(\operatorname{Hom}_{\Lambda}(M, \Lambda), \Lambda\right)$. By [V1, Proposition 2.5] and the Auslander condition, the canonical map $\phi: M \longrightarrow M^{++}$has kernel and cokernel which are $\Lambda$-torsion. Therefore, $\phi$ induces an isomorphism

$$
Q(\Lambda) \otimes_{\Lambda} M \stackrel{\sim}{\longrightarrow} Q(\Lambda) \otimes_{\Lambda} M^{++} .
$$

Now if $\phi=0$, then it will follow that $Q(\Lambda) \otimes_{\Lambda} M=0$. This establishes (a) $\Rightarrow(\mathrm{b})$.

Therefore, if $G$ is pro- $p$ and has no $p$-torsion, it follows from the above lemma that a finitely generated $\mathcal{O} \llbracket G \rrbracket$-module $M$ is torsion if and only if $\operatorname{Hom}_{\mathcal{O} \llbracket G \rrbracket}(M, \mathcal{O} \llbracket G \rrbracket)=0$. Now if $M$ is a finitely generated torsion $\mathcal{O} \llbracket G \rrbracket$-module, we say that $M$ is pseudo-null if $\operatorname{Ext}_{\mathcal{O} \llbracket G \rrbracket}^{1}(M, \mathcal{O} \llbracket G \rrbracket)=0$. For an equivalent definition, we refer readers to [V1, Definitions 3.1 and 3.3; Proposition 3.5(ii)]. For the purpose of this article, the definition we adopt will suffice. Finally, we mention that every subquotient of a torsion $\mathcal{O} \llbracket G \rrbracket$-module (resp., pseudo-null $\mathcal{O} \llbracket G \rrbracket$-module) is also torsion (resp. pseudo-null).

\section{$2.3 \mu$-invariant}

Let $\mathcal{O}$ be the ring of integers of a fixed finite extension $K$ of $\mathbb{Q}_{p}$ as defined in the preceding subsection. Fix a local parameter $\pi$ for $\mathcal{O}$ and denote the residue field of $\mathcal{O}$ by $k$. The completed group algebra of $G$ over $k$ is given by

$$
k \llbracket G \rrbracket=\varliminf_{U}^{\lim } k[G / U],
$$

where $U$ runs over the open normal subgroups of $G$ and the inverse limit is taken with respect to the canonical projection maps.

For a compact $p$-adic Lie group $G$ without $p$-torsion, it follows from [V1, Theorem 3.30(ii)] that $k \llbracket G \rrbracket$ is an Auslander regular ring. Furthermore, if $G$ is pro- $p$ without $p$-torsion, then the ring $k \llbracket G \rrbracket$ has no zero divisors (cf. $[\mathrm{AB}$, Theorem $\mathrm{C}]$ ). Therefore, one can define the notion of $k \llbracket G \rrbracket$-rank as above when $G$ is pro- $p$ without $p$-torsion. We will say that that the module $N$ is a torsion $k \llbracket G \rrbracket$-module if $\operatorname{rank}_{k \llbracket G \rrbracket} N=0$. By Lemma 2.2.1, we have that $N$ is a torsion $k \llbracket G \rrbracket$-module if and only if $\operatorname{Hom}_{k \llbracket G \rrbracket}(N, k \llbracket G \rrbracket)=0$.

For a given finitely generated $\mathcal{O} \llbracket G \rrbracket$-module $M$, we denote $M(\pi)$ to be the $\mathcal{O} \llbracket G \rrbracket$-submodule of $M$ which consists of elements of $M$ that are annihilated by some power of $\pi$. Since the ring $\mathcal{O} \llbracket G \rrbracket$ is Noetherian, the module $M(\pi)$ is finitely generated over $\mathcal{O} \llbracket G \rrbracket$. Therefore, one can find an integer $r \geq 0$ such that $\pi^{r}$ annihilates $M(\pi)$. Following [Ho1, Formula (33)], we define

$$
\mu_{\mathcal{O} \llbracket G \rrbracket}(M)=\sum_{i \geq 0} \operatorname{rank}_{k \llbracket G \rrbracket}\left(\pi^{i} M(\pi) / \pi^{i+1}\right) .
$$


(For another alternative, but equivalent, definition, see [V1, Definition 3.32].) By the above discussion and our definition of $k \llbracket G \rrbracket$-rank, the sum on the right is a finite one. It is clear from the definition that $\mu_{\mathcal{O} \llbracket G \rrbracket}(M)=\mu_{\mathcal{O} \llbracket G \rrbracket}(M(\pi))$. Also, it is not difficult to see that this definition coincides with the classical notion of the $\mu$-invariant for $\Gamma$-modules when $G=\Gamma \cong \mathbb{Z}_{p}$.

We now record certain properties of the $\mu_{\mathcal{O} \llbracket G \rrbracket}$-invariant which will be required in the subsequent of the paper.

Lemma 2.3.1. Let $G$ be a compact pro-p p-adic Lie group with no $p$-torsion and let $M$ be a finitely generated $\mathcal{O} \llbracket G \rrbracket$-module. Then we have the following statements.

(a) For every finitely generated $\mathcal{O} \llbracket G \rrbracket$-module $M$, one has

$$
\mu_{G}(M)=\sum_{i \geq 0}(-1)^{i} \operatorname{ord}_{q}\left(H_{i}(G, M(\pi))\right)
$$

where $q$ is the cardinality of $k$.

(b) Suppose that $G$ has a closed normal subgroup $H$ such that $G / H \cong \mathbb{Z}_{p}$. If $M$ is a $\mathcal{O} \llbracket G \rrbracket$ module which is finitely generated over $\mathcal{O} \llbracket H \rrbracket$, then one has $\mu_{\mathcal{O} \llbracket G \rrbracket}(M)=0$.

(c) Suppose that we are given a short exact sequence of finitely generated $\mathcal{O} \llbracket G \rrbracket$ modules

$$
0 \longrightarrow M^{\prime} \longrightarrow M \longrightarrow M^{\prime \prime} \longrightarrow 0
$$

(1) One has $\mu_{\mathcal{O} \llbracket G \rrbracket}(M) \leq \mu_{\mathcal{O} \llbracket G \rrbracket}\left(M^{\prime}\right)+\mu_{\mathcal{O} \llbracket G \rrbracket}\left(M^{\prime \prime}\right)$. Moreover, if $M$, and hence also $M^{\prime}$ and $M^{\prime \prime}$, is $\mathcal{O} \llbracket G \rrbracket$-torsion, the inequality is an equality.

(2) If $\mu_{\mathcal{O} \llbracket G \rrbracket}\left(M^{\prime \prime}\right)=0$, then one has $\mu_{\mathcal{O} \llbracket G \rrbracket}\left(M^{\prime}\right)=\mu_{\mathcal{O} \llbracket G \rrbracket}(M)$.

(d) Suppose that $G$ has a closed normal subgroup $H$ such that $G / H \cong \mathbb{Z}_{p}$ and suppose that we are given an exact sequence of finitely generated $\mathcal{O} \llbracket G \rrbracket$-modules

$$
A \longrightarrow B \longrightarrow C \longrightarrow D
$$

such that $A$ is finitely generated over $\mathcal{O} \llbracket H \rrbracket$ and $\mu_{G}(D)=0$. Then one has the equality $\mu_{G}(B)=\mu_{G}(C)$.

(e) $\mu_{\mathcal{O} \llbracket G \rrbracket}(M)=0$ if and only if $M(\pi)$ is pseudo-null.

Proof. Statements (a), (b) and (c)(1) are proven in [Ho1, Corollary 1.7], [Ho1, Lemma 2.7] and [Ho1, Proposition 1.8] respectively. Statement (e) is shown in [V1, Remark 3.33]. The remaining statements can be deduced from the previous statements without too much difficulties. 


\section{$2.4 \pi$-primary modules}

Throughout this subsection, $G$ will always denote a pro- $p$ p-adic Lie group without $p$-torsion. Therefore, both rings $\mathcal{O} \llbracket G \rrbracket$ and $k \llbracket G \rrbracket$ are Auslander regular and have no zero divisors. For a finitely generated $\mathcal{O} \llbracket G \rrbracket$-module $M$, it then follows from [Ho2, Proposition 1.11] (see also [V1, Theorem 3.40]) that there is a $\mathcal{O} \llbracket G \rrbracket$-homomorphism

$$
\varphi: M(\pi) \longrightarrow \bigoplus_{i=1}^{s} \mathcal{O} \llbracket G \rrbracket / \pi^{\alpha_{i}}
$$

whose kernel and cokernel are pseudo-null $\mathcal{O} \llbracket G \rrbracket$-modules, and where the integers $s$ and $\alpha_{i}$ are uniquely determined. We will call $\bigoplus_{i=1}^{s} \mathcal{O} \llbracket G \rrbracket / \pi^{\alpha_{i}}$ the elementary representation of $M(\pi)$. In fact, in the process of establishing the above, one also has that $\mu_{\mathcal{O} \llbracket G \rrbracket}(M)=\sum_{i=1}^{s} \alpha_{i}$ (see loc. cit.). We will set

$$
\theta_{\mathcal{O} \llbracket G \rrbracket}(M):=\max _{1 \leq i \leq s}\left\{\alpha_{i}\right\} .
$$

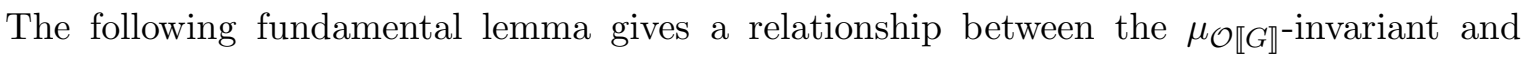
$\mathcal{O} \llbracket G \rrbracket$-rank of a finitely generated $\mathcal{O} \llbracket G \rrbracket$-module.

Lemma 2.4.1. Let $M$ be a finitely generated $\mathcal{O} \llbracket G \rrbracket$-module. Suppose that there is a $\mathcal{O} \llbracket G \rrbracket$ homomorphism

$$
\varphi: M(\pi) \longrightarrow \bigoplus_{i=1}^{s} \mathcal{O} \llbracket G \rrbracket / \pi^{\alpha_{i}}
$$

whose kernel and cokernel are pseudo-null $\mathcal{O} \llbracket G \rrbracket$-modules. Then we have

$$
\mu_{\mathcal{O} \llbracket G \rrbracket}\left(M / \pi^{n}\right)=n \operatorname{rank}_{\mathcal{O} \llbracket G \rrbracket}(M)+\sum_{i=1}^{s} \min \left\{n, \alpha_{i}\right\} \quad \text { for } n \geq 1 .
$$

In particular, we have $\mu_{\mathcal{O} \llbracket G \rrbracket}\left(M / \pi^{n}\right) \leq n \operatorname{rank}_{\mathcal{O} \llbracket G \rrbracket}(M)+\mu_{\mathcal{O} \llbracket G \rrbracket}(M)$ which is an equality if and only if $n \geq \theta_{\mathcal{O} \llbracket G \rrbracket}(M)$.

Proof. Write $M_{f}=M / M(\pi)$. Consider the following commutative diagram

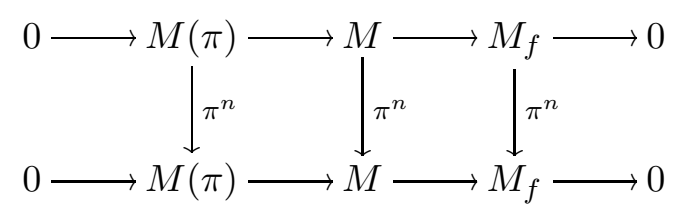

with exact rows, and the vertical maps are given by multiplication by $\pi^{n}$. Since $M_{f}$ has no $\pi$-torsion, the rightmost vertical map is injective, and therefore, we have an exact sequence

$$
0 \longrightarrow M(\pi) / \pi^{n} \longrightarrow M / \pi^{n} \longrightarrow M_{f} / \pi^{n} \longrightarrow 0
$$


of torsion $\mathcal{O} \llbracket G \rrbracket$-modules. By Lemma 2.3.1(c)(1), we have

$$
\mu_{\mathcal{O} \llbracket G \rrbracket}\left(M / \pi^{n}\right)=\mu_{\mathcal{O} \llbracket G \rrbracket}\left(M(\pi) / \pi^{n}\right)+\mu_{\mathcal{O} \llbracket G \rrbracket}\left(M_{f} / \pi^{n}\right) .
$$

To prove the lemma, it therefore suffices to show the following two equalities.

(1) $\mu_{\mathcal{O} \llbracket G \rrbracket}\left(M(\pi) / \pi^{n}\right)=\sum_{i=1}^{s} \min \left\{n, \alpha_{i}\right\}$.

(2) $\mu_{\mathcal{O} \llbracket G \rrbracket}\left(M_{f} / \pi^{n}\right)=n \operatorname{rank}_{\mathcal{O} \llbracket G \rrbracket}\left(M_{f}\right)=n \operatorname{rank}_{\mathcal{O} \llbracket G \rrbracket}(M)$.

To see that (1) holds, note that since any subquotient of a pseudo-null module is also pseudonull, it follows that $\varphi$ induces an $\mathcal{O} \llbracket G \rrbracket$-homomorphism

$$
M(\pi) / \pi^{n} \longrightarrow \bigoplus_{i=1}^{s} \mathcal{O} \llbracket G \rrbracket / \pi^{\min \left\{n, \alpha_{i}\right\}},
$$

whose kernel and cokernel are pseudo-null $\mathcal{O} \llbracket G \rrbracket$-modules. The equality in (1) will now follow by combining this observation with statements (c)(1) and (e) of Lemma 2.3.1.

Since $M(\pi)$ is clearly a torsion $\mathcal{O} \llbracket G \rrbracket$-module, we have $\operatorname{rank}_{\mathcal{O} \llbracket G \rrbracket}\left(M_{f}\right)=\operatorname{rank}_{\mathcal{O} \llbracket G \rrbracket}(M)$. Therefore, it remains to verify the first equality in (2). In other words, we are reduced to showing that if $M$ is a finitely generated $\mathcal{O} \llbracket G \rrbracket$-module with $M(\pi)=0$, then $\mu_{\mathcal{O} \llbracket G \rrbracket}\left(M / \pi^{n}\right)=n \operatorname{rank}_{\mathcal{O} \llbracket G \rrbracket}(M)$. We shall proceed by induction. Suppose that $n=1$. Then we have

$$
\operatorname{rank}_{\mathcal{O} \llbracket G \rrbracket}(M)=\operatorname{rank}_{k \llbracket G \rrbracket}(M / \pi)=\sum_{i \geq 0}(-1)^{i} \operatorname{dim}_{k}\left(H_{i}(G, M / \pi)\right)=\mu_{\mathcal{O} \llbracket G \rrbracket}(M / \pi),
$$

where the first equality follows from [Ho1, Corollary 1.10] and the assumption that $M[\pi]=0$, the second equality follows from [Ho1, Proposition 1.6], and the third equality follows from Lemma 2.3.1(a). Therefore, we have established the $n=1$ case.

Now suppose that $n>1$, and suppose that $\mu_{\mathcal{O} \llbracket G \rrbracket}\left(M / \pi^{n-1}\right)=(n-1) \operatorname{rank}_{\mathcal{O} \llbracket G \rrbracket}(M)$. Then consider the following commutative diagram

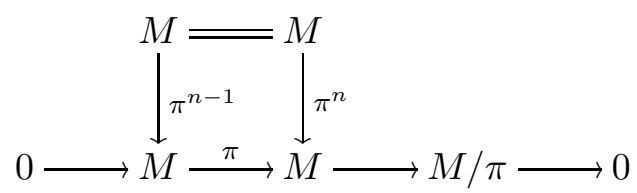

with exact bottom row (note that injectivity follows from the assumption that $M[\pi]=0$ ). By the snake lemma, we have an exact seqeuence

$$
0 \longrightarrow M / \pi^{n-1} \longrightarrow M / \pi^{n} \longrightarrow M / \pi \longrightarrow 0
$$

of torsion $\mathcal{O} \llbracket G \rrbracket$-modules which in turn yields

$$
\begin{aligned}
\mu_{\mathcal{O} \llbracket G \rrbracket}\left(M / \pi^{n}\right) & =\mu_{\mathcal{O} \llbracket G \rrbracket}(M / \pi)+\mu_{\mathcal{O} \llbracket G \rrbracket}\left(M / \pi^{n-1}\right) \\
& =\operatorname{rank}_{\mathcal{O} \llbracket G \rrbracket}(M)+(n-1) \operatorname{rank}_{\mathcal{O} \llbracket G \rrbracket}(M)=n \operatorname{rank}_{\mathcal{O} \llbracket G \rrbracket}(M) .
\end{aligned}
$$

The proof of the lemma is completed. 
Remark 2.4.2. When $G \cong \mathbb{Z}_{p}^{r}$, one can prove the above lemma by appealing directly to the structure theory (cf. [NSW, Proposition 5.1.7]).

We record the following lemma which enables one to relate $M(\pi)$ and $N(\pi)$ in certain situation.

Lemma 2.4.3. Suppose that $H$ is a closed normal subgroup of $G$ with $G / H \cong \mathbb{Z}_{p}$. Let $\varphi: M \longrightarrow$ $N$ be a homomorphism of finitely generated $\mathcal{O} \llbracket G \rrbracket$-modules, whose kernel and cokernel are finitely generated over $\mathcal{O} \llbracket H \rrbracket$. Then $M(\pi)$ and $N(\pi)$ have the same elementary representations.

Proof. The statement will follow if it holds in the two special cases of exact sequences

$$
\begin{aligned}
& 0 \longrightarrow P \longrightarrow M \longrightarrow N \longrightarrow 0, \\
& 0 \longrightarrow M \longrightarrow N \longrightarrow P \longrightarrow 0,
\end{aligned}
$$

where $P$ is a finitely generated $\mathcal{O} \llbracket H \rrbracket$-module. We will prove the first case, the second case has a similar argument. Choose a sufficiently large $n$ such that $\pi^{n}$ annihilates $M(\pi)$ and $N(\pi)$. Consider the following commutative diagram

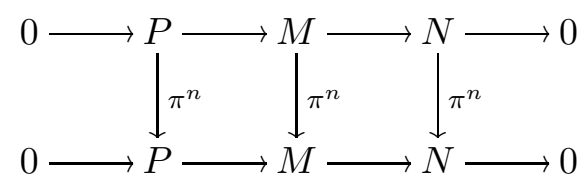

with exact rows, and the vertical maps are given by multiplication by $\pi^{n}$. Applying the Snake Lemma, we obtain

$$
0 \longrightarrow P\left[\pi^{n}\right] \longrightarrow M(\pi) \longrightarrow N(\pi) \longrightarrow P / \pi^{n}
$$

By Lemma 2.3.1(b) and (e), we have that $P\left[\pi^{n}\right]$ and $P / \pi^{n}$ are pseudo-null $\mathcal{O} \llbracket G \rrbracket$-modules. Let

$$
f: N(\pi) \longrightarrow \bigoplus_{i=1}^{s} \mathcal{O} \llbracket G \rrbracket / \pi^{\alpha_{i}}
$$

be a homomorphism of $\mathcal{O} \llbracket G \rrbracket$-modules, whose kernel and cokernel are pseudo-null $\mathcal{O} \llbracket G \rrbracket$-modules. Then

$$
f \circ \varphi: M(\pi) \longrightarrow \bigoplus_{i=1}^{s} \mathcal{O} \llbracket G \rrbracket / \pi^{\alpha_{i}}
$$

is a homomorphism of $\mathcal{O} \llbracket G \rrbracket$-modules, whose kernel and cokernel are pseudo-null. Therefore, $M(\pi)$ and $N(\pi)$ have the same elementary representations.

Proposition 2.4.4. Let $M$ and $N$ be two finitely generated torsion $\mathcal{O} \llbracket G \rrbracket$-modules such that $\mu_{\mathcal{O} \llbracket G \rrbracket}\left(M / \pi^{\theta_{\mathcal{O} \llbracket G \rrbracket}(M)}\right)=\mu_{\mathcal{O} \llbracket G \rrbracket}\left(N / \pi^{\theta_{\mathcal{O} \llbracket G \rrbracket}(M)}\right)$. Then we have

$$
\mu_{\mathcal{O} \llbracket G \rrbracket}(M) \leq \mu_{\mathcal{O} \llbracket G \rrbracket}(N) .
$$


Proof. By Lemma 2.4.1, we have

$$
\mu_{\mathcal{O} \llbracket G \rrbracket}(M)=\mu_{\mathcal{O} \llbracket G \rrbracket}\left(M / \pi^{\theta_{\mathcal{O} \llbracket G \rrbracket}(M)}\right)=\mu_{\mathcal{O} \llbracket G \rrbracket}\left(N / \pi^{\theta_{\mathcal{O} \llbracket G \rrbracket}(M)}\right) \leq \mu_{\mathcal{O} \llbracket G \rrbracket}(N) .
$$

Now for a given finitely generated torsion $\mathcal{O} \llbracket G \rrbracket$-module $M$, the elementary representation of $M(\pi)$ can be rewritten as

$$
\bigoplus_{i=1}^{\theta}\left(\mathcal{O} \llbracket G \rrbracket / \pi^{i}\right)^{s_{i}}
$$

for some nonnegative integers $s_{i}$. Here $\theta=\theta_{\mathcal{O} \llbracket G \rrbracket}(M)$. Then for every $1 \leq n \leq \theta$, we have

$$
\mu_{\mathcal{O} \llbracket G \rrbracket}\left(M / \pi^{n}\right)=s_{1}+2 s_{2}+\cdots+(n-1) s_{n-1}+n\left(s_{n}+\cdots s_{\theta}\right) .
$$

Putting these equations into a matrix form, we have

$$
\left(\begin{array}{c}
\mu_{\mathcal{O} \llbracket G \rrbracket}(M / \pi) \\
\mu_{\mathcal{O} \llbracket G \rrbracket}\left(M / \pi^{2}\right) \\
\mu_{\mathcal{O} \llbracket G \rrbracket}\left(M / \pi^{3}\right) \\
\vdots \\
\mu_{\mathcal{O} \llbracket G \rrbracket}\left(M / \pi^{\theta}\right)
\end{array}\right)=\left(\begin{array}{ccccc}
1 & 1 & 1 & \cdots & 1 \\
1 & 2 & 2 & \cdots & 2 \\
1 & 2 & 3 & \cdots & 3 \\
\vdots & \vdots & \vdots & \ddots & \vdots \\
1 & 2 & 3 & \cdots & \theta
\end{array}\right)\left(\begin{array}{c}
s_{1} \\
s_{2} \\
s_{3} \\
\vdots \\
s_{\theta}
\end{array}\right) .
$$

It is a simple linear algebra exercise to verify that the square matrix in the above equation is invertible. Therefore, the integers $s_{i}$, and hence the elementary representation of $M(\pi)$, are determined by the values of $\mu_{\mathcal{O} \llbracket G \rrbracket}\left(M / \pi^{i}\right)$. We record this observation in the next proposition.

Proposition 2.4.5. Let $M$ and $N$ be two finitely generated torsion $\mathcal{O} \llbracket G \rrbracket$-modules. Then the following are equivalent.

(a) $\theta_{\mathcal{O} \llbracket G \rrbracket}(M)=\theta_{\mathcal{O} \llbracket G \rrbracket}(N)$, and $\mu_{\mathcal{O} \llbracket G \rrbracket}\left(M / \pi^{i}\right)=\mu_{\mathcal{O} \llbracket G \rrbracket}\left(N / \pi^{i}\right)$ for every $1 \leq i \leq \theta_{\mathcal{O} \llbracket G \rrbracket}(M)$.

(b) $M(\pi)$ and $N(\pi)$ have the same elementary representations.

Proof. The discussion before the proposition establishes the implication $(a) \Rightarrow(b)$. The reverse implication is obvious.

The preceding proposition may be difficult to apply due to the condition $\theta_{\mathcal{O} \llbracket G \rrbracket}(M)=$ $\theta_{\mathcal{O} \llbracket G \rrbracket}(N)$ which is perhaps not easy to check. However, one can build on the proposition to obtain the following which is perhaps easier for application. 
Proposition 2.4.6. Let $M$ and $N$ be two finitely generated $\mathcal{O} \llbracket G \rrbracket$-modules such that $M$ is a torsion $\mathcal{O} \llbracket G \rrbracket$-module and such that $\mu_{\mathcal{O} \llbracket G \rrbracket}\left(M / \pi^{i}\right)=\mu_{\mathcal{O} \llbracket G \rrbracket}\left(N / \pi^{i}\right)$ for every $1 \leq i \leq \theta_{\mathcal{O} \llbracket G \rrbracket}(M)+1$.

Then $N$ is torsion over $\mathcal{O} \llbracket G \rrbracket$ and we have the equality $\theta_{\mathcal{O} \llbracket G \rrbracket}(M)=\theta_{\mathcal{O} \llbracket G \rrbracket}(N)$. In particular, $M(\pi)$ and $N(\pi)$ have the same elementary representations.

Proof. We first prove the proposition for the case when $\theta_{\mathcal{O} \llbracket G \rrbracket}(M)=0$. Then by the hypothesis of the proposition, we have $\mu_{\mathcal{O} \llbracket G \rrbracket}(N / \pi)=\mu_{\mathcal{O} \llbracket G \rrbracket}(M / \pi)=0$. By Lemma 2.4.1, this in turns implies that $\operatorname{rank}_{\mathcal{O} \llbracket G \rrbracket}(N)=0$ and $\mu_{\mathcal{O} \llbracket G \rrbracket}(N)=0$. Therefore, we have that $N$ is torsion over $\mathcal{O} \llbracket G \rrbracket$ and $\theta_{\mathcal{O} \llbracket G \rrbracket}(N)=0$. Hence we have that $M(\pi)$ and $N(\pi)$ are both pseudo-null by Lemma 2.3.1(e), and therefore, have the same elementary representations.

Now suppose that $\theta_{\mathcal{O} \llbracket G \rrbracket}(M) \geq 1$. Suppose that the elementary factor of $N(\pi)$ is given by

$$
\bigoplus_{i=1}^{t} \mathcal{O} \llbracket G \rrbracket / \pi^{\beta_{i}}
$$

Write $a=\operatorname{rank}_{\mathcal{O} \llbracket G \rrbracket}(N)$. By Lemma 2.4.1, we then have

$$
\mu_{\mathcal{O} \llbracket G \rrbracket}(M)=\mu_{\mathcal{O} \llbracket G \rrbracket}\left(M / \pi^{n}\right)=\mu_{\mathcal{O} \llbracket G \rrbracket}\left(N / \pi^{n}\right)=n a+\sum_{i=1}^{t} \min \left\{n, \beta_{i}\right\}
$$

for $n=\theta_{\mathcal{O} \llbracket G \rrbracket}(M), \theta_{\mathcal{O} \llbracket G \rrbracket}(M)+1$. This in turn implies that

$$
\theta_{\mathcal{O} \llbracket G \rrbracket}(M) a+\sum_{i=1}^{t} \min \left\{\theta_{\mathcal{O} \llbracket G \rrbracket}(M), \beta_{i}\right\}=\left(\theta_{\mathcal{O} \llbracket G \rrbracket}(M)+1\right) a+\sum_{i=1}^{t} \min \left\{\theta_{\mathcal{O} \llbracket G \rrbracket}(M)+1, \beta_{i}\right\} .
$$

Since one always has $\theta_{\mathcal{O} \llbracket G \rrbracket}(M) a \leq\left(\theta_{\mathcal{O} \llbracket G \rrbracket}(M)+1\right) a$ and $\min \left\{\theta_{\mathcal{O} \llbracket G \rrbracket}(M), \beta_{i}\right\} \leq \min \left\{\theta_{\mathcal{O} \llbracket G \rrbracket}(M)+\right.$ $\left.1, \beta_{i}\right\}$, in order for the above equality to hold, we must have $a=0$ and $\min \left\{\theta_{\mathcal{O} \llbracket G \rrbracket}(M), \beta_{i}\right\}=$ $\min \left\{\theta_{\mathcal{O} \llbracket G \rrbracket}(M)+1, \beta_{i}\right\}$ for $1 \leq i \leq t$. The formal equality then shows that $N$ is a torsion $\mathcal{O} \llbracket G \rrbracket$-module, and the latter equalities show that $\beta_{i} \leq \theta_{\mathcal{O} \llbracket G \rrbracket}(M)$ for all $i$, or in other words, $\theta_{\mathcal{O} \llbracket G \rrbracket}(N) \leq \theta_{\mathcal{O} \llbracket G \rrbracket}(M)$. Therefore, we may repeat the above argument (noting that we have shown that $N$ is $\mathcal{O} \llbracket G \rrbracket$-torsion) replacing $\theta_{\mathcal{O} \llbracket G \rrbracket}(M)$ by $\theta_{\mathcal{O} \llbracket G \rrbracket}(N)$ and interchanging the roles of $M$ and $N$ to obtain the reverse inequality $\theta_{\mathcal{O} \llbracket G \rrbracket}(M) \leq \theta_{\mathcal{O} \llbracket G \rrbracket}(N)$. The remaining assertion will now follow from an application of Proposition 2.4.5.

In particular, it follows from Proposition 2.4.6 that if $M$ and $N$ are two finitely generated torsion $\mathcal{O} \llbracket G \rrbracket$-modules such that $\mu_{\mathcal{O} \llbracket G \rrbracket}\left(M / \pi^{i}\right)=\mu_{\mathcal{O} \llbracket G \rrbracket}\left(N / \pi^{i}\right)$ for every $i \geq 1$, then $M(\pi)$ and $N(\pi)$ have the same elementary representations. In fact, we can even establish the following stronger statement. 
Proposition 2.4.7. Let $M$ and $N$ be two finitely generated $\mathcal{O} \llbracket G \rrbracket$-modules such that $\mu_{\mathcal{O} \llbracket G \rrbracket}\left(M / \pi^{i}\right)=$ $\mu_{\mathcal{O} \llbracket G \rrbracket}\left(N / \pi^{i}\right)$ for every $i \geq 1$.

Then we have that $\operatorname{rank}_{\mathcal{O} \llbracket G \rrbracket}(M)=\operatorname{rank}_{\mathcal{O} \llbracket G \rrbracket}(N)$ and that $M(\pi)$ and $N(\pi)$ have the same elementary representations.

Proof. For $n \geq \max \left\{\theta_{\mathcal{O} \llbracket G \rrbracket}(M), \theta_{\mathcal{O} \llbracket G \rrbracket}(N)\right\}$, it follows from the assumption $\mu_{\mathcal{O} \llbracket G \rrbracket}\left(M / \pi^{n}\right)=$ $\mu_{\mathcal{O} \llbracket G \rrbracket}\left(N / \pi^{n}\right)$ and Lemma 2.4.1 that

$$
n \operatorname{rank}_{\mathcal{O} \llbracket G \rrbracket}(M)+\mu_{\mathcal{O} \llbracket G \rrbracket}(M)=n \operatorname{rank}_{\mathcal{O} \llbracket G \rrbracket}(N)+\mu_{\mathcal{O} \llbracket G \rrbracket}(N) .
$$

In other words, we have

$$
\operatorname{rank}_{\mathcal{O} \llbracket G \rrbracket}(M)+\frac{1}{n} \mu_{\mathcal{O} \llbracket G \rrbracket}(M)=\operatorname{rank}_{\mathcal{O} \llbracket G \rrbracket}(N)+\frac{1}{n} \mu_{\mathcal{O} \llbracket G \rrbracket}(N) .
$$

Letting $n \rightarrow \infty$, we obtain

$$
\operatorname{rank}_{\mathcal{O} \llbracket G \rrbracket}(M)=\operatorname{rank}_{\mathcal{O} \llbracket G \rrbracket}(N) .
$$

This proves the first assertion.

As seen in the proof of Lemma 2.4.1, we have

$$
\mu_{\mathcal{O} \llbracket G \rrbracket}\left(M / \pi^{i}\right)=\mu_{\mathcal{O} \llbracket G \rrbracket}\left(M(\pi) / \pi^{i}\right)+i \operatorname{rank}_{\mathcal{O} \llbracket G \rrbracket}(M) .
$$

One has a similar equality for $N$. It then follows from these equalities and what we proved in the preceding paragraph that

$$
\mu_{\mathcal{O} \llbracket G \rrbracket}\left(M(\pi) / \pi^{i}\right)=\mu_{\mathcal{O} \llbracket G \rrbracket}\left(N(\pi) / \pi^{i}\right)
$$

for all $i \geq 1$. The second assertion will now follow from an application of Proposition 2.4.6 on $M(\pi)$ and $N(\pi)$.

\subsection{An asymptotic formula}

In this subsection, $G$ will always denote a pro- $p$-adic Lie group without $p$-torsion. We denote by $r$ the dimension of $G$. We fix an open normal uniform subgroup $G_{0}$ of $G$ (such a group exists by virtue of Lazard's theorem [Laz]). In the event that $G$ is already a uniform group, we take $G_{0}=G$. We now write $G_{m}$ for $P_{m+1}\left(G_{0}\right)$ which is defined as in Subsection 2.1. As before, $\mathcal{O}$ is the ring of integers of a finite extension $K$ of $\mathbb{Q}_{p}, \pi$ is a local parameter of $\mathcal{O}$ and $k$ is the residue field of $\mathcal{O}$. Denote $q$ to the order of $k$. Every finite $\mathcal{O}$-module can be viewed as a $\mathcal{O} / \pi^{n}$-module for some $n$. Since $\mathcal{O} / \pi^{n}$ has order of a power of $q$, so is every finite $\mathcal{O}$-module. For a finite $\mathcal{O}$-module, we will denote $\operatorname{ord}_{q}(M)$ to be the exponent of $q$ in the order of $M$, i.e., $|M|=q^{\operatorname{ord}_{q}(M)}$. 
We take this opportunity to introduce a notion which will used in this paper. A sequence of real numbers $\left(a_{m}\right)_{m \geq 1}$ is said to satisfy $O\left(Q^{m}\right)$ for some nonnegative number $Q$ if $\left|a_{m}\right| \leq C Q^{m}$ for some constant $C$ (independent of $m$ ) for all sufficiently large $m$. We will write $a_{m}=O\left(Q^{m}\right)$. If $\left(b_{m}\right)_{m \geq 1}$ is another sequence of real numbers, we will write $a_{m}=b_{m}+O\left(Q^{m}\right)$ to mean $a_{m}-b_{m}=O\left(Q^{m}\right)$.

We can now state the main theorem of this subsection.

Theorem 2.5.1. Let $G$ be pro-p p-adic Lie group without $p$-torsion. Write $r=\operatorname{dim} G$. Let $M$ be a finitely generated $\mathcal{O} \llbracket G \rrbracket$-module such that $M=M(\pi)$. Then we have

$$
\operatorname{ord}_{q}\left(M_{G_{m}}\right)=\left[G: G_{0}\right] \mu_{\mathcal{O} \llbracket G \rrbracket}(M) p^{r m}+O\left(p^{(r-1) m}\right)
$$

and

$$
\operatorname{ord}_{q}\left(H_{i}\left(G_{m}, M\right)\right)=O\left(p^{(r-1) m}\right)
$$

for every $i \geq 1$.

Remark 2.5.2. The first asymptotic formula in the above result is a weak analog of the asymptotic formula of Iwasawa [Iw, Theorem 4] (see also [NSW, Proposition 5.3.17]). When $G=\mathbb{Z}_{p}^{r}$, this can also be viewed as a weak analog of the asymptotic formula of Cucuo and Monsky [CM, Theorem 4.13] (see also [Mon, Theorem 3.12]).

It seems possible that the above formula might be known among the experts. Despite so, due to a lack of proper reference, we will include a proof here. (In fact, as we shall see, the tools required for the proof are available from [Har1, Har2, Ho1, Ho2, V1].) For our purpose in this paper, we will only require the first asymptotic formula. Despite so, we have included the proof of the asymptotic formulas for the higher cohomology groups for completeness.

The proof of Theorem 2.5.1 will take up the remainder of this subsection. As a start, we note that $\left[G: G_{0}\right] \mu_{\mathcal{O} \llbracket G \rrbracket}(M)=\mu_{\mathcal{O} \llbracket G_{0} \rrbracket}(M)$. Since a $\mathcal{O} \llbracket G \rrbracket$-module can be viewed as a $\mathcal{O} \llbracket G_{0} \rrbracket$ by restriction of scalars, it suffices to prove the theorem under the assumption that $G$ is uniform. In view of this, we therefore can and do assume that $G$ is uniform for the subsequent of this subsection. For the preparation of the proof, we require a few lemmas.

Lemma 2.5.3. Let $M$ be a finitely generated torsion $k \llbracket G \rrbracket-m o d u l e$. Then

$$
\operatorname{ord}_{q}\left(M_{G_{m}}\right)=O\left(p^{(r-1) m}\right)
$$

Proof. Since $M$ is finitely generated torsion over $k \llbracket G \rrbracket$, there is a surjective map

$$
\bigoplus_{j} k \llbracket G \rrbracket / k \llbracket G \rrbracket f_{j} \longrightarrow M
$$


for a finite set of non-zero and non-unital elements $f_{i} \in k \llbracket G \rrbracket$. Therefore, one is reduced to the case $M=k \llbracket G \rrbracket / k \llbracket G \rrbracket f$ for some non-zero and non-unital $f$. The remainder of the proof then proceeds as in the proofs of [Har1, Lemma 1.10.1] and [Har2, Theorem 1.10], where one passes to the graded ring of $k \llbracket G \rrbracket$ and appeals to the theory of Hilbert polynomials.

We will also require an estimate for $\operatorname{ord}_{q}\left(H_{i}\left(G_{m}, M\right)\right)$. Before showing this, we need the following lemma.

Lemma 2.5.4. Let $f$ be a nonzero nonunital element of $k \llbracket G \rrbracket$, and set $M=k \llbracket G \rrbracket / k \llbracket G \rrbracket f$. Then for every $m$, we have

$$
\operatorname{ord}_{q}\left(H_{1}\left(G_{m}, M\right)\right)=\operatorname{ord}_{q}\left(M_{G_{m}}\right)
$$

and $H_{i}\left(G_{m}, M\right)=0$ for $i \geq 2$.

Proof. Since $k \llbracket G \rrbracket$ has no zero divisors, we have an exact sequence

$$
0 \longrightarrow k \llbracket G \rrbracket \stackrel{\cdot f}{\longrightarrow} k \llbracket G \rrbracket \longrightarrow M \longrightarrow 0 .
$$

Since $H_{i}\left(G_{m}, k \llbracket G \rrbracket\right)=0$ for $i \geq 1$, it follows from considering the $G_{m}$-homology that we obtain an exact sequence

$$
0 \longrightarrow H_{1}\left(G_{m}, M\right) \longrightarrow k\left[G / G_{m}\right] \longrightarrow k\left[G / G_{m}\right] \longrightarrow M_{G_{m}} \longrightarrow 0
$$

and the vanishing of $H_{i}\left(G_{m}, M\right)$ for $i \geq 2$. The first conclusion of the lemma is now immediate from the four term exact sequence.

We can now give an estimate for $\operatorname{ord}_{q}\left(H_{i}\left(G_{m}, M\right)\right)$.

Lemma 2.5.5. Let $M$ be a finitely generated torsion $k \llbracket G \rrbracket$-module. Then for every $i \geq 1$, we have

$$
\operatorname{ord}_{q}\left(H_{i}\left(G_{m}, M\right)\right)=O\left(p^{(r-1) m}\right),
$$

where $r$ denotes the dimension of $G$.

Proof. As above, we have a exact sequence

$$
0 \longrightarrow N \longrightarrow \bigoplus_{j} k \llbracket G \rrbracket / k \llbracket G \rrbracket f_{j} \longrightarrow M \longrightarrow 0
$$

of torsion $k \llbracket G \rrbracket$-modules. Taking the $G_{m}$-homology, we have an exact sequence

$$
H_{i}\left(G_{m}, \bigoplus_{j} k \llbracket G \rrbracket / k \llbracket G \rrbracket f_{j}\right) \longrightarrow H_{i}\left(G_{m}, M\right) \longrightarrow H_{i-1}\left(G_{m}, N\right) .
$$

The required estimates will follow from the previous two lemmas. 
We now establish our estimates for the case when $M=M(\pi)$ is a finitely generated pseudo-

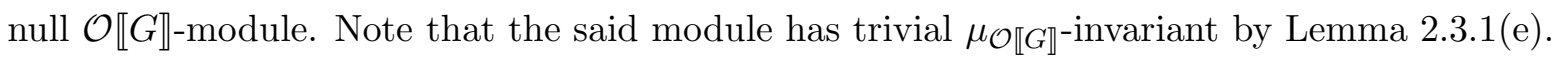

Lemma 2.5.6. Let $G$ be a uniform pro-p group of dimension $r$. Suppose that $M=M(\pi)$ is a finitely generated pseudo-null $\mathcal{O} \llbracket G \rrbracket$-module. Then for each $i \geq 0$, we have

$$
\operatorname{ord}_{q}\left(H_{i}\left(G_{m}, M\right)\right)=O\left(p^{(r-1) m}\right) .
$$

Proof. Since $M$ is annihilated by a power of $\pi$, it is a finite successive extension of subquotients $\pi^{i} M / \pi^{i+1}$. Therefore, it suffices to bound each of these subquotients. Since subquotients of a pseudo-null $\mathcal{O} \llbracket G \rrbracket$-module are also pseudo-null, we are reduced to showing that if $M$ is a finitely generated pseudo-null $\mathcal{O} \llbracket G \rrbracket$-module with $\pi M=0$, then $\operatorname{ord}_{q}\left(M_{G_{m}}\right)=O\left(p^{(r-1) m}\right)$. Since $\pi M=0$, we may also view $M$ as a $k \llbracket G \rrbracket$-module. By a standard spectral sequence argument (for instance, see [V1, Section 3.4]), we have

$$
\operatorname{Ext}_{k \llbracket G \rrbracket}^{i}(M, k \llbracket G \rrbracket) \cong \operatorname{Ext}_{\mathcal{O} \llbracket G \rrbracket}^{i+1}(M, \mathcal{O} \llbracket G \rrbracket)
$$

for any integer $i$. In particular, we have

$$
\operatorname{Hom}_{k \llbracket G \rrbracket}(M, k \llbracket G \rrbracket) \cong \operatorname{Ext}_{\mathcal{O} \llbracket G \rrbracket}^{1}(M, \mathcal{O} \llbracket G \rrbracket)=0,
$$

where the last equality follows from the fact that $M$ is pseudo-null over $\mathcal{O} \llbracket G \rrbracket$. Hence $M$ is a torsion $k \llbracket G \rrbracket$-module. The first estimate then follows from Lemma 2.5.3, The estimates for the higher cohomology groups can be proven similarly making use of Lemma 2.5.5.

We record one more lemma.

Lemma 2.5.7. Suppose that $M$ and $N$ are two finitely generated $\mathcal{O} \llbracket G \rrbracket$-modules with $M=M(\pi)$ and $N=N(\pi)$. Assume that there is a $\mathcal{O} \llbracket G \rrbracket$-homomorphism $\varphi: M \longrightarrow N$ which has pseudonull kernel and cokernel. Then for $i \geq 0$, we have

$$
\operatorname{ord}_{q}\left(H_{i}\left(G_{m}, M\right)\right)=\operatorname{ord}_{q}\left(H_{i}\left(G_{m}, N\right)\right)+O\left(p^{(r-1) m}\right)
$$

Proof. The statement will follow if it holds in the two special cases of exact sequences

$$
\begin{aligned}
& 0 \longrightarrow M \longrightarrow N \longrightarrow P \longrightarrow 0, \\
& 0 \longrightarrow P \longrightarrow M \longrightarrow N \longrightarrow 0,
\end{aligned}
$$

where $P$ is a pseudo-null $\mathcal{O} \llbracket G \rrbracket$-module. Note that $P=P(\pi)$. Taking $G_{m}$-homology of the first exact sequence, we have

$$
H_{1}\left(G_{m}, P\right) \longrightarrow M_{G_{m}} \longrightarrow N_{G_{m}} \longrightarrow P_{G_{m}} \longrightarrow 0
$$


By Lemmas 2.5.3 and 2.5.5, we have

$$
\operatorname{ord}_{q}\left(H_{1}\left(G_{m}, P\right)\right)=\operatorname{ord}_{q}\left(P_{G_{m}}\right)=O\left(q^{(r-1) m}\right) .
$$

The cases for the higher cohomology groups and for the second exact sequence can be proven similarly.

We can now prove our theorem.

Proof of Theorem 2.5.1. Let

$$
\varphi: M \longrightarrow \bigoplus_{i=1}^{s} \mathcal{O} \llbracket G \rrbracket / \pi^{\alpha_{i}}
$$

be a $\mathcal{O} \llbracket G \rrbracket$-homomorphism whose kernel and cokernel are pseudo-null (recall that we are assuming $M=M(\pi))$. By Lemma 2.5.7, we have

$$
\operatorname{ord}_{q}\left(H_{i}\left(G_{m}, M\right)\right)=\sum_{i=1}^{s} \operatorname{ord}_{q}\left(H_{i}\left(G_{m}, \mathcal{O} \llbracket G \rrbracket / \pi^{\alpha_{i}}\right)\right)+O\left(p^{(r-1) m}\right) .
$$

Therefore, we are reduced to showing that

$$
\operatorname{ord}_{q}\left(H_{i}\left(G_{m}, \mathcal{O} \llbracket G \rrbracket / \pi^{\alpha}\right)\right)= \begin{cases}\alpha p^{r m} & \text { if } i=0 \\ 0 & \text { if } i \geq 1 .\end{cases}
$$

Since $\mathcal{O} \llbracket G \rrbracket$ has no zero divisors, we have an exact sequence

$$
0 \longrightarrow \mathcal{O} \llbracket G \rrbracket \stackrel{\cdot \pi^{\alpha}}{\longrightarrow} \mathcal{O} \llbracket G \rrbracket \longrightarrow \mathcal{O} \llbracket G \rrbracket / \pi^{\alpha} \longrightarrow 0 .
$$

Since $H_{i}\left(G_{m}, \mathcal{O} \llbracket G \rrbracket\right)=0$ for $i \geq 1$, it follows from considering the $G_{m}$-homology that

$$
H_{i}\left(G_{m}, \mathcal{O} \llbracket G \rrbracket / \pi^{\alpha}\right)=0
$$

for $i \geq 1$. It remains to show the first equality, and this is immediate from the facts that $\mathcal{O}\left[G / G_{m}\right] / \pi^{\alpha} \cong\left(\mathcal{O} / \pi^{\alpha}\right)^{p^{r m}}$ (as abelian groups) and that $\left|\mathcal{O} / \pi^{\alpha}\right|=q^{\alpha}$.

\section{Arithmetic Preliminaries}

In this section, we introduce the Selmer groups and Selmer complexes. Here, we fix the notation that we shall use throughout this section. To start, let $p$ be a prime. We let $F$ be a number field. If $p=2$, we assume further that $F$ has no real primes. Denote $\mathcal{O}$ to be the ring of integers of some finite extension $K$ of $\mathbb{Q}_{p}$, and fix a local parameter $\pi$ for $\mathcal{O}$. Suppose that we are given the following datum $\left(A,\left\{A_{v}\right\}_{v \mid p},\left\{A_{v}^{+}\right\}_{v \mid \mathbb{R}}\right)$ defined over $F$ : 
(a) $A$ is a cofinitely generated cofree $\mathcal{O}$-module of $\mathcal{O}$-corank $d$ with a continuous, $\mathcal{O}$-linear $\operatorname{Gal}(\bar{F} / F)$-action which is unramified outside a finite set of primes of $F$.

(b) For each prime $v$ of $F$ above $p, A_{v}$ is a $\operatorname{Gal}\left(\bar{F}_{v} / F_{v}\right)$-submodule of $A$ which is cofree of $\mathcal{O}$-corank $d_{v}$.

(c) For each real prime $v$ of $F$, we write $A_{v}^{+}=A^{\mathrm{Gal}\left(\bar{F}_{v} / F_{v}\right)}$ which is assumed to be cofree of $\mathcal{O}$-corank $d_{v}^{+}$.

(d) The following equality

$$
\sum_{v \mid p}\left(d-d_{v}\right)\left[F_{v}: \mathbb{Q}_{p}\right]=d r_{2}(F)+\sum_{v \text { real }}\left(d-d_{v}^{+}\right)
$$

holds. Here $r_{2}(F)$ denotes the number of complex primes of $F$.

We now consider the base change property of our datum. Let $L$ be a finite extension of $F$. We can then obtain another datum $\left(A,\left\{A_{w}\right\}_{w \mid p},\left\{A_{w}^{+}\right\}_{w \mid \mathbb{R}}\right)$ over $L$ as follows: we consider $A$ as a $\operatorname{Gal}(\bar{F} / L)$-module, and for each prime $w$ of $L$ above $p$, we set $A_{w}=A_{v}$, where $v$ is a prime of $F$ below $w$, and view it as a $\operatorname{Gal}\left(\bar{F}_{v} / L_{w}\right)$-module. Then $d_{w}=d_{v}$. For each real prime $w$ of $L$, one sets $A^{\operatorname{Gal}\left(\bar{L}_{w} / L_{w}\right)}=A^{\operatorname{Gal}\left(\bar{F}_{v} / F_{v}\right)}$ and writes $d_{w}^{+}=d_{v}^{+}$, where $v$ is a real prime of $F$ below $w$. In general, the $d_{w}$ 's and $d_{w}^{+}$'s need not satisfy equality (3.0.1). We now record the following lemma which gives some sufficient conditions for equality (3.0.1) to hold for the datum $\left(A,\left\{A_{w}\right\}_{w \mid p},\left\{A_{w}^{+}\right\}_{w \mid \mathbb{R}}\right)$ over $L$.

Lemma 3.0.1. Suppose that $\left(A,\left\{A_{v}\right\}_{v \mid p},\left\{A_{v}^{+}\right\}_{v \mid \mathbb{R}}\right)$ is a datum defined over $F$. Suppose further that at least one of the following statements holds.

(i) All the archimedean primes of $F$ are unramified in $L$.

(ii) $[L: F]$ is odd

(iii) $F$ is totally imaginary.

(iv) $F$ is totally real, $L$ is totally imaginary and

$$
\sum_{v \text { real }} d_{v}^{+}=d[F: Q] / 2
$$

Then we have the equality

$$
\sum_{w \mid p}\left(d-d_{w}\right)\left[L_{w}: \mathbb{Q}_{p}\right]=d r_{2}(L)+\sum_{w \text { real }}\left(d-d_{w}^{+}\right)
$$


Proof. Note that if either of the assertions in (ii) or (iii) holds, then the assertion in (i) holds. Therefore, to prove the lemma in these cases, it suffices to prove it under the assumption of (i). We first perform the following calculation

$$
\begin{aligned}
\sum_{w \mid p}\left(d-d_{w}\right)\left[L_{w}: \mathbb{Q}_{p}\right] & =\sum_{v \mid p} \sum_{w \mid v}\left(d-d_{v}\right)\left[L_{w}: F_{v}\right]\left[F_{v}: \mathbb{Q}_{p}\right] \\
& =\sum_{v \mid p}\left(d-d_{v}\right)\left[F_{v}: \mathbb{Q}_{p}\right] \sum_{w \mid v}\left[L_{w}: F_{v}\right] \\
& =[L: F] \sum_{v \mid p}\left(d-d_{v}\right)\left[F_{v}: \mathbb{Q}_{p}\right] \\
& =d[L: F] r_{2}(F)+[L: F] \sum_{v \text { real }}\left(d-d_{v}^{+}\right) .
\end{aligned}
$$

Now if (i) holds, then every prime of $L$ above a real prime (resp., complex prime) of $F$ is a real prime (resp., complex prime). Therefore, one has $[L: F] r_{2}(F)=r_{2}(L)$ and

$$
[L: F] \sum_{v \text { real }}\left(d-d_{v}^{+}\right)=\sum_{w \text { real }}\left(d-d_{w}^{+}\right)
$$

The required conclusion then follows.

Now suppose that (iv) holds. Then $r_{2}(F)=0$ and we have

$$
\begin{aligned}
\sum_{w \mid p}\left(d-d_{w}\right)\left[L_{w}: \mathbb{Q}_{p}\right]=[L: F] \sum_{v \text { real }}\left(d-d_{v}^{+}\right) & =[L: F] \sum_{v \text { real }} d-[L: F] \sum_{v \text { real }} d_{v}^{+} \\
& =[L: F][F: \mathbb{Q}] d-[L: F] d[F: \mathbb{Q}] / 2 \\
& =d[L: \mathbb{Q}] / 2=d r_{2}(L) .
\end{aligned}
$$

We now describe briefly the arithmetic situation, where we can obtain the above set of data from. Let $V$ be a $d$-dimensional $K$-vector space with a continuous $\operatorname{Gal}(\bar{F} / F)$-action which is unramified outside a finite set of primes. Suppose that for each prime $v$ of $F$ above $p$, there is a $d_{v}$-dimensional $K$-subspace $V_{v}$ of $V$ which is invariant under the action of $\operatorname{Gal}\left(\bar{F}_{v} / F_{v}\right)$, and for each real prime $v$ of $F, V^{\operatorname{Gal}\left(\bar{F}_{v} / F_{v}\right)}$ has dimension $d_{v}^{+}$. Choose a $\operatorname{Gal}(\bar{F} / F)$-stable $\mathcal{O}$-lattice $T$ of $V$ (Such a lattice exists by compactness). We can obtain a data as above from $V$ by setting $A=V / T$ and $A_{v}=V_{v} / T \cap V_{v}$. Note that $A$ and $A_{v}$ depends on the choice of the lattice $T$. We mention some concrete examples.

(1) Let $B$ be an abelian variety of dimension $g$ defined over a number field $F$. For simplicity, we assume that $F$ is totally imaginary and that the abelian variety $B$ has semistable reduction over $F$. We define a set of data $\left(A,\left\{A_{v}\right\}\right)$ by first setting $A=B\left[p^{\infty}\right]$. For each prime $v$ 
of $F$ above $p$, let $\mathcal{F}_{v}$ be the formal group attached to the Neron model for $B$ over the ring of integers $\mathcal{O}_{F_{v}}$ of $F_{v}$, and we assume that $\mathcal{F}_{v}$ is a formal group of height $g$ for all $v \mid p$. For instance, this latter condition is satisfied if $B$ has good ordinary reduction at all $v \mid p$. We then set $A_{v}=\mathcal{F}_{v}(\overline{\mathfrak{m}})\left[p^{\infty}\right]$, where $\overline{\mathfrak{m}}$ is the maximal ideal of the rings of integers of $\bar{F}_{v}$. Note that $A_{v} \cong\left(\mathbb{Q}_{p} / \mathbb{Z}_{p}\right)^{g}$ as an abelian group by our height assumption. It is easy to see that $\left(A,\left\{A_{v}\right\}\right)$ satisfies the condition in Section 3 by taking $d=2 g$ and $d_{v}=g$. It is worthwhile mentioning that the (strict) Selmer groups attached to this set of data coincide with the classical Selmer groups of the abelian variety, when the Selmer groups are considered over an admissible $p$-adic Lie extension (see [CG]).

(2) More generally, a source of examples where we can obtain such a datum is that of a nearly ordinary Galois representation in the sense of Weston We]. This is a finite-dimensional $K$-vector space equipped with a $K$-linear action of the absolute Galois group $\operatorname{Gal}(\bar{F} / F)$ such that for each prime $v$ of $F$ dividing $p$, there is sequence

$$
0=V_{v, 0} \subseteq V_{v, 1} \subseteq \cdots \subseteq V_{v, n}=V
$$

of nearly ordinary $\operatorname{Gal}\left(\bar{F}_{v} / F_{v}\right)$-subspace of $V$, where $V_{v, i}$ has $K$-dimension $i$. Following $[\mathrm{We}$, a set of Selmer weights for $V$ is a choice of integers $c_{v}\left(0 \leq c_{v} \leq d\right)$ for each $v$ dividing $p$ such that

$$
\sum_{v \mid p} c_{v}\left[F_{v}: \mathbb{Q}_{p}\right]=d r_{2}(F)+\sum_{v \text { real }}\left(d-d_{v}^{+}\right)
$$

where $r_{2}(F)$ denotes the number of complex primes of $F$. Set $V_{v}=V_{v, d-c_{v}}$. (In other words, our $d_{v}$ is $d-c_{v}$.) For more concrete examples, we refer readers to [Gr1, §9] and [We, Section $1.2]$.

\subsection{Selmer groups}

We now introduce the Selmer groups. Let $S$ be a finite set of primes of $F$ which contains all the primes above $p$, the ramified primes of $A$ and all the infinite primes of $F$. Denote $F_{S}$ to be the maximal algebraic extension of $F$ unramified outside $S$ and write $G_{S}(\mathcal{L})=\operatorname{Gal}\left(F_{S} / \mathcal{L}\right)$ for every algebraic extension $\mathcal{L}$ of $F$ which is contained in $F_{S}$. Let $L$ be a finite extension of $F$ contained in $F_{S}$ such that the data $\left(A,\left\{A_{w}\right\}_{w \mid p},\left\{A_{w}^{+}\right\}_{w \mid \mathbb{R}}\right)$ satisfies (3.0.1). For a prime $w$ of $L$ lying over $S$, set

$$
H_{s t r}^{1}\left(L_{w}, A\right)= \begin{cases}\operatorname{ker}\left(H^{1}\left(L_{w}, A\right) \longrightarrow H^{1}\left(L_{w}, A / A_{w}\right)\right) & \text { if } w \text { divides } p \\ \operatorname{ker}\left(H^{1}\left(L_{w}, A\right) \longrightarrow H^{1}\left(L_{w}^{u r}, A\right)\right) & \text { if } w \text { does not divide } p\end{cases}
$$


where $L_{w}^{u r}$ is the maximal unramified extension of $L_{w}$. The (strict) Selmer group attached to the data is then defined by

$$
S(A / L):=\operatorname{Sel}^{s t r}(A / L):=\operatorname{ker}\left(H^{1}\left(G_{S}(L), A\right) \longrightarrow \bigoplus_{w \in S_{L}} H_{s}^{1}\left(L_{w}, A\right)\right),
$$

where we write $H_{s}^{1}\left(L_{w}, A\right)=H^{1}\left(L_{w}, A\right) / H_{s t r}^{1}\left(L_{w}, A\right)$ and $S_{L}$ denotes the set of primes of $L$ above $S$. It is straightforward to verify that $S(A / L)=\underset{n}{\lim } S\left(A\left[\pi^{n}\right] / L\right)$, where $S\left(A\left[\pi^{n}\right] / L\right)$ is the Selmer group defined similarly as above by replacing $A$ by $A\left[\pi^{n}\right]$ and $A_{v}$ by $A_{v}\left[\pi^{n}\right]$. Here the direct limit is taken over the maps $S\left(A\left[\pi^{n}\right] / L\right) \longrightarrow S\left(A\left[\pi^{n+1}\right] / L\right)$ which are induced by the natural injections $A\left[\pi^{n}\right] \hookrightarrow A\left[\pi^{n+1}\right]$ and $A_{w}\left[\pi^{n}\right] \hookrightarrow A_{w}\left[\pi^{n+1}\right]$. We will write $X(A / L)$ for its Pontryagin dual.

We shall say that $F_{\infty}$ is an $S$-admissible $p$-adic Lie extension of $F$ if (i) $\operatorname{Gal}\left(F_{\infty} / F\right.$ ) is compact $p$-adic Lie group, (ii) $F_{\infty}$ contains the cyclotomic $\mathbb{Z}_{p}$ extension $F_{\text {cyc }}$ of $F$ and (iii) $F_{\infty}$ is unramified outside $S$. Write $G=\operatorname{Gal}\left(F_{\infty} / F\right), H=\operatorname{Gal}\left(F_{\infty} / F_{\text {cyc }}\right)$ and $\Gamma=\operatorname{Gal}\left(F_{\text {cyc }} / F\right)$. We define $S\left(A / F_{\infty}\right)=\underset{L}{\lim } S(A / L)$, where the limit runs over all finite extensions $L$ of $F$ contained in $F_{\infty}$. We write $X\left(A / F_{\infty}\right)$ for the Pontryagin dual of $S\left(A / F_{\infty}\right)$. By a similar argument to that in [CS, Corollary 2.3], one can show that $X\left(A / F_{\infty}\right)$ is independent of the choice of $S$ as long as $S$ contains all the primes above $p$, the ramified primes of $A$, the primes that ramify in $F_{\infty} / F$ and all infinite primes.

We introduce another variant of the Selmer groups which is usually called the Greenberg Selmer group. Now set

$$
H_{G r}^{1}\left(F_{v}, A\right)= \begin{cases}\operatorname{ker}\left(H^{1}\left(F_{v}, A\right) \longrightarrow H^{1}\left(F_{v}^{u r}, A / A_{v}\right)\right) & \text { if } v \mid p, \\ \operatorname{ker}\left(H^{1}\left(F_{v}, A\right) \longrightarrow H^{1}\left(F_{v}^{u r}, A\right)\right) & \text { if } v \nmid p .\end{cases}
$$

The Greenberg Selmer group attached to these data is then defined by

$$
\operatorname{Sel}^{G r}(A / F)=\operatorname{ker}\left(H^{1}\left(G_{S}(F), A\right) \longrightarrow \bigoplus_{v \in S} H_{g}^{1}\left(F_{v}, A\right)\right),
$$

where we write $H_{g}^{1}\left(F_{v}, A\right)=H^{1}\left(F_{v}, A\right) / H_{G r}^{1}\left(F_{v}, A\right)$. For an $S$-admissible $p$-adic Lie extension $F_{\infty}$, we define $\operatorname{Sel}^{G r}\left(A / F_{\infty}\right)=\underset{L}{\lim } \operatorname{Sel}^{G r}(A / L)$ and denote $X^{G r}\left(A / F_{\infty}\right)$ to be the Pontryagin dual of $\operatorname{Sel}^{G r}\left(A / F_{\infty}\right)$. The following lemma compares the two Selmer groups of Greenberg.

Lemma 3.1.1. We have an exact sequence

$$
0 \longrightarrow S\left(A / F_{\infty}\right) \longrightarrow \operatorname{Sel}^{G r}\left(A / F_{\infty}\right) \longrightarrow N \longrightarrow 0
$$

where $N$ is a cofinitely generated $\mathcal{O} \llbracket H \rrbracket$-module. 
Proof. Now consider the following commutative diagram

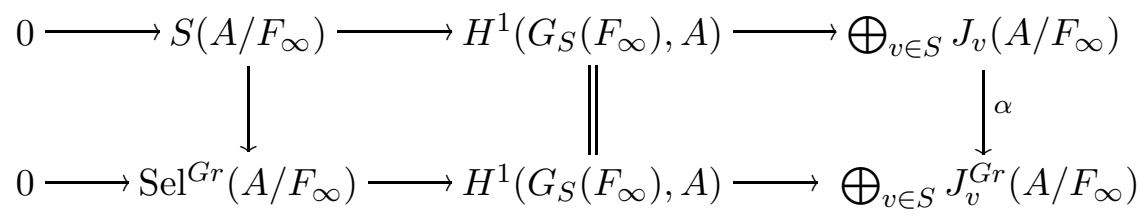

with exact rows, where $J_{v}^{G r}\left(A / F_{\infty}\right)=\underset{L}{\lim } \bigoplus_{w \mid v} H_{g}^{1}\left(L_{w}, A\right)$. It therefore remains to show that ker $\alpha$ is cofinitely generated over $\mathcal{O} \llbracket H \rrbracket$. Clearly, $J_{v}\left(A / F_{\infty}\right)=J_{v}^{G r}\left(A / F_{\infty}\right)$ for $v \nmid p$. For each $v \mid p$, fix a prime $w$ of $F_{\infty}$ above $v$. Write $I_{\infty, w}$ for the inertia subgroup of $\operatorname{Gal}\left(\bar{F}_{\infty, w} / F_{\infty, w}\right)$ and $U_{w}=\operatorname{Gal}\left(\bar{F}_{\infty, w} / F_{\infty, w}\right) / I_{\infty, w}$. It then follows from the Hochschild-Serre spectral sequence that we have

$$
0 \longrightarrow H^{1}\left(U_{w},\left(A / A_{v}\right)^{I_{\infty, w}}\right) \longrightarrow H^{1}\left(H_{w}, A / A_{v}\right) \longrightarrow H^{1}\left(I_{\infty, w}, A / A_{v}\right)^{U_{w}}
$$

Since $U_{w}$ is topologically cyclic, $H^{1}\left(U_{w},\left(A / A_{v}\right)^{I_{\infty, w}}\right) \cong\left(\left(A / A_{v}\right)^{I_{\infty}, w}\right)_{U_{v}}$ and so is cofinitely generated over $\mathcal{O}$. Since $F_{\infty}$ contains $F^{\text {cyc }}$, the decomposition group of $G$ at $v$ has at least dimension one for each $v \mid p$. Hence it follows that ker $\alpha$ is cofinitely generated over $\mathcal{O} \llbracket H \rrbracket$, as required.

Lemma 3.1.2. One has

$$
\operatorname{rank}_{\mathcal{O} \llbracket G \rrbracket}\left(X\left(A / F_{\infty}\right)\right)=\operatorname{rank}_{\mathcal{O} \llbracket G \rrbracket}\left(X^{G r}\left(A / F_{\infty}\right)\right)
$$

and $X\left(A / F_{\infty}\right)(\pi)$ and $X^{G r}\left(A / F_{\infty}\right)(\pi)$ have the same elementary representations.

Proof. By the preceding lemma, one has an exact sequence

$$
0 \longrightarrow N^{\prime} \longrightarrow X^{G r}\left(A / F_{\infty}\right) \longrightarrow X\left(A / F_{\infty}\right) \longrightarrow 0
$$

for some finitely generated $\mathcal{O} \llbracket H \rrbracket$-module $N^{\prime}$. The first equality is immediate, and the second assertion follows from Lemma 2.4.3.

\subsection{Selmer complexes}

We now consider the Selmer complex associated to the data $\left(A,\left\{A_{v}\right\}_{v \mid p},\left\{A_{v}^{+}\right\}_{v \mid \mathbb{R}}\right)$. The notion of a Selmer complex was first conceived and introduced in [Nek]. In our discussion, we consider a modified version of the Selmer complex as given in [FK, 4.2.11]. Write $T^{*}=\operatorname{Hom}_{\mathrm{cts}}\left(A, \mu_{p^{\infty}}\right)$ and $T_{v}^{*}=\operatorname{Hom}_{\mathrm{cts}}\left(A / A_{v}, \mu_{p^{\infty}}\right)$. For every finite extension $L$ of $F$ and $w$ a prime of $L$ above $p$, write 
$T_{w}^{*}=T_{v}^{*}$, where $v$ is the prime of $F$ below $w$. For any profinite group $\mathcal{G}$ and a topological abelian group $M$ with a continuous $\mathcal{G}$-action, we denote by $C(\mathcal{G}, M)$ the complex of continuous cochains of $\mathcal{G}$ with coefficients in $M$. Let $F_{\infty}$ be an $S$-admissible extension of $F$ with Galois group $G$. We define a $(\mathcal{O} \llbracket G \rrbracket)\left[G_{S}(F)\right]$-module $\mathcal{F}_{G}\left(T^{*}\right)$ as follows: as an $\mathcal{O}$-module, $\mathcal{F}_{G}\left(T^{*}\right)=\mathcal{O} \llbracket G \rrbracket \otimes_{\mathcal{O}} T^{*}$, and the action of $G_{S}(F)$ is given by the formula $\sigma(x \otimes t)=x \bar{\sigma}^{-1} \otimes \sigma t$, where $\bar{\sigma}$ is the canonical image of $\sigma$ in $G \subseteq \mathcal{O} \llbracket G \rrbracket$. We define the $(\mathcal{O} \llbracket G \rrbracket)\left[\operatorname{Gal}\left(\bar{F}_{v} / F_{v}\right)\right]$-module $\mathcal{F}_{G}\left(T_{v}^{*}\right)$ in a similar fashion.

For every prime $v$ of $F$, we write $C\left(F_{v}, \mathcal{F}_{G}\left(T^{*}\right)\right)=C\left(\operatorname{Gal}\left(\bar{F}_{v} / F_{v}\right), \mathcal{F}_{G}\left(T^{*}\right)\right)$. For each prime $v$ not dividing $p$, denote $C_{f}\left(F_{v}, \mathcal{F}_{G}\left(T^{*}\right)\right)$ to be the subcomplex of $C\left(F_{v}, \mathcal{F}_{G}\left(T^{*}\right)\right)$, whose degree $m$-component is 0 unless $m \neq 0,1$, whose degree 0 -component is $C^{0}\left(F_{v}, \mathcal{F}_{G}\left(T^{*}\right)\right)$, and whose degree 1-component is

$$
\operatorname{ker}\left(C^{1}\left(F_{v}, \mathcal{F}_{G}\left(T^{*}\right)\right)_{d=0} \longrightarrow H^{1}\left(F_{v}^{u r}, \mathcal{F}_{G}\left(T^{*}\right)\right)\right) .
$$

The Selmer complex $S C\left(T^{*}, T_{v}^{*}\right)$ is then defined to be

Cone $\left(C\left(G_{S}(F), \mathcal{F}_{G}\left(T^{*}\right)\right) \longrightarrow \bigoplus_{v \mid p} C\left(F_{v}, \mathcal{F}_{G}\left(T^{*}\right) / \mathcal{F}_{G}\left(T_{v}^{*}\right)\right) \oplus \bigoplus_{v \nmid p} C\left(F_{v}, \mathcal{F}_{G}\left(T^{*}\right)\right) / C_{f}\left(F_{v}, \mathcal{F}_{G}\left(T^{*}\right)\right)\right)[-1]$.

Here $[-1]$ is the translation by -1 of the complex. We will write $H^{i}\left(S C\left(T^{*}, T_{v}^{*}\right)\right)$ for the $i$ th cohomology group of the complex $S C\left(T^{*}, T_{v}^{*}\right)$. We now state the following proposition which is proven in [FK, Proposition 4.2.35].

Proposition 3.2.1. Let $\mathcal{G}$ be the kernel of $\operatorname{Gal}(\bar{F} / F) \longrightarrow G$. For a place $v$ of $F$, fixing an embedding $F \hookrightarrow F_{v}$, let $\mathcal{G}(v)$ be the kernel of $\operatorname{Gal}\left(\bar{F}_{v} / F_{v}\right) \longrightarrow G$ and let $G_{v} \subseteq G$ be the image. Then the following statements hold.

(a) $H^{i}\left(S C\left(T^{*}, T_{v}^{*}\right)\right)=0$ for $i \neq 1,2,3$.

(b) We have an exact sequence

$$
\begin{aligned}
0 \longrightarrow X\left(A / F_{\infty}\right) \longrightarrow H^{2}\left(S C\left(T^{*}, T_{v}^{*}\right)\right) & \longrightarrow \bigoplus_{v \mid p} \mathcal{O} \llbracket G \rrbracket \otimes_{\mathcal{O} \llbracket G_{v} \rrbracket}\left(T_{v}^{*}(-1)\right)_{\mathcal{G}(v)} \\
& \longrightarrow\left(T^{*}(-1)\right)_{\mathcal{G}} \longrightarrow H^{3}\left(S C\left(T^{*}, T_{v}^{*}\right)\right) \longrightarrow 0
\end{aligned}
$$

Since $F_{\infty}$ contains $F_{\text {cyc }}$, it follows that for every prime $v \mid p$, the group $G_{v}$ has dimension at least 1. Therefore, $\bigoplus_{v \mid p} \mathcal{O} \llbracket G \rrbracket \otimes_{\mathcal{O} \llbracket G_{v} \rrbracket}\left(T_{v}^{*}(-1)\right)_{\mathcal{G}(v)}$ is finitely generated over $\mathcal{O} \llbracket H \rrbracket$, and one can apply Lemma 2.4.3 to obtain the following statement.

Lemma 3.2.2. $X\left(A / F_{\infty}\right)(\pi)$ and $H^{2}\left(S C\left(T^{*}, T_{v}^{*}\right)\right)(\pi)$ have the same elementary representations. 
We end the section with the following remark.

Remark 3.2.3. It is clear from the exact sequence in Proposition 3.2 .1 that $H^{3}\left(S C\left(T^{*}, T_{v}^{*}\right)\right)$ is finitely generated over $\mathcal{O}$. In particular, this implies that $H^{3}\left(S C\left(T^{*}, T_{v}^{*}\right)\right)(\pi)$ is pseudo-null. Now assume further that $X\left(A / F_{\infty}\right)$ satisfies the $\mathfrak{M}_{H}(G)$-property in the sense of $\left[\mathrm{CF}^{+}, \mathrm{CS}\right.$, FK] (see also Subsection [5.1). Note that when $F_{\infty}$ is of dimension 1, this is equivalent to saying that $X\left(A / F_{\infty}\right)$ is a torsion module. Then it follows from [FK, Proposition 4.3.11] that $H^{1}\left(S C\left(T^{*}, T_{v}^{*}\right)=0\right.$ (resp., $H^{1}\left(S C\left(T^{*}, T_{v}^{*}\right)\right.$ is finitely generated over $\left.\mathcal{O}\right)$ if $F_{\infty}$ is of dimension $>1$ (resp., dimension 1). In either cases, we have that $H^{1}\left(S C\left(T^{*}, T_{v}^{*}\right)\right)(\pi)$ is pseudo-null. Therefore, it follows from the above discussion, Proposition 3.2.1 (a) and Lemma 3.2.2 that the $\pi$-primary submodule of $X\left(A / F_{\infty}\right)$ essentially captures the " $\pi$-primary component" of the Selmer complex $S C\left(T^{*}, T_{v}^{*}\right)$.

\section{$4 \pi$-submodules of dual Selmer groups}

Throughout this section, $\left(A,\left\{A_{v}\right\}_{v \mid p},\left\{A_{v}^{+}\right\}_{v \mid \mathbb{R}}\right)$ is a datum defined as in Section 3 over a number field $F$. As before, $S$ will denote a finite set of primes that contain the primes of $F$ above $p$, the ramified primes of $A$ and the archimedean primes of $F$. Let $F_{\infty}$ be an $S$-admissible $p$-adic Lie extension of $F$ whose Galois group $G=\operatorname{Gal}\left(F_{\infty} / F\right)$ is a pro- $p$ torsion-free $p$-adic Lie group of dimension $r$. We also recall that $G_{0}$ is a fixed open normal uniform subgroup of $G$, and $G_{m}$ is denoted to be the $(m+1)$-term of the lower $p$-series of $G_{0}$ which is defined by

$$
G_{m+1}=G_{m}^{p}\left[G_{m}, G\right], \text { for } m \geq 0 .
$$

Denote $F_{m}$ to be the fixed field of $G_{m}$. Note that this is a finite Galois extension of $F$ of degree $\left[G: G_{0}\right] p^{r m}$.

For the remainder of the paper, we will work with $X\left(A / F_{\infty}\right)$. In view of Lemmas 3.1.2 and 3.2.2, all our main results (Theorem 4.1.1 and Theorem 4.2.1) also hold for the Greenberg Selmer groups and the second cohomology of the Selmer complexes as defined in Subsection 3.2 ,

If $G$ is a pro- $p$ group, we write $h_{1}(G)=\operatorname{dim}_{\mathbb{Z} / p}\left(H^{1}(G, \mathbb{Z} / p)\right)$ and $h_{2}(G)=\operatorname{dim}_{\mathbb{Z} / p}\left(H^{2}(G, \mathbb{Z} / p)\right)$. If $M$ is a cofinitely generated $\mathcal{O}$-module, we denote $M_{\text {div }}$ to be the maximal $\mathcal{O}$-divisible submodule of $M$. We now record a useful lemma which allows us to estimate the order of certain cohomology groups, whose easy proof is left to the reader.

Lemma 4.0.1. Let $G$ be a pro-p group, and let $M$ be a discrete $G$-module which is cofinitely generated over $\mathcal{O}$. Let $n$ be a positive integer. If $h_{1}(G)$ is finite, then for every $n \geq 1, H^{1}(G, M)\left[\pi^{n}\right]$ is finite and

$$
\operatorname{ord}_{q}\left(H^{1}(G, M)\left[\pi^{n}\right]\right) \leq n h_{1}(G)\left(\operatorname{corank}_{\mathcal{O}}(M)+\operatorname{ord}_{q}\left(M / M_{\text {div }}\right)\right)
$$


If $h_{2}(G)$ is finite, then for every $n \geq 1, H^{2}(G, M)\left[\pi^{n}\right]$ is finite and we have the following inequality

$$
\operatorname{ord}_{q}\left(H^{2}(G, M)\left[\pi^{n}\right]\right) \leq n h_{2}(G)\left(\operatorname{corank}_{\mathcal{O}}(M)+\operatorname{ord}_{q}\left(M / M_{\text {div }}\right)\right)
$$

\subsection{Tate dual}

In this subsection, we prove our first main result of the paper. For a given set of data $\left(A,\left\{A_{v}\right\}_{v \mid p},\left\{A_{v}^{+}\right\}_{v \mid \mathbb{R}}\right)$, we define its (Tate) dual data as follows. For a $\mathcal{O}$-module $N$, we denote $T_{\pi}(N)$ to be its $\pi$-adic Tate module, i.e., $T_{\pi}(N)=\underset{\varliminf_{i}}{\lim } N\left[\pi^{i}\right]$. We then set $A^{*}=$ $\operatorname{Hom}_{\mathrm{cts}}\left(T_{\pi}(A), \mu_{p^{\infty}}\right)$. Similarly, for each $v \mid p$ (resp., $v$ real), we set $A_{v}^{*}=\operatorname{Hom}_{\mathrm{cts}}\left(T_{\pi}\left(A / A_{v}\right), \mu_{p^{\infty}}\right)$ (resp., $\left.\left(A^{*}\right)_{v}^{+}=\operatorname{Hom}_{\mathrm{cts}}\left(T_{\pi}\left(A / A_{v}^{+}\right), \mu_{p^{\infty}}\right)\right)$. It is an easy exercise to verify that $\left(A^{*},\left\{A_{v}^{*}\right\}_{v \mid p},\left\{\left(A^{*}\right)_{v}^{+}\right\}_{v \mid \mathbb{R}}\right)$ satisfies equality (3.0.1). Therefore, we can attach Selmer groups to this dual data which we denote by $S\left(A^{*} / F_{\infty}\right)$ and $S\left(A^{*}\left[\pi^{n}\right] / F_{\infty}\right)$. We then denote $X\left(A^{*} / F_{\infty}\right)$ to be the Pontryagin dual of $S\left(A^{*} / F_{\infty}\right)$. We are now in the position to state the first main theorem of the paper.

Theorem 4.1.1. Let $F_{\infty}$ be an admissible p-adic Lie extension such that $G=\operatorname{Gal}\left(F_{\infty} / F\right)$ is pro-p torsion-free $p$-adic Lie group. Then $X\left(A / F_{\infty}\right)$ and $X\left(A^{*} / F_{\infty}\right)$ have the same $\mathcal{O} \llbracket G \rrbracket$-ranks, and $X\left(A / F_{\infty}\right)(\pi)$ and $X\left(A^{*} / F_{\infty}\right)(\pi)$ have the same elementary representations.

For data coming from (nearly) ordinary representations, it is expected that $X\left(A / F_{\infty}\right)$ is a torsion $\mathcal{O} \llbracket G \rrbracket$-module (see [Gr1, Conjecture 1] or [We, Conjecture 1.7]). We therefore record the following important corollary.

Corollary 4.1.2. Let $F_{\infty}$ be an admissible $p$-adic Lie extension such that $G=\operatorname{Gal}\left(F_{\infty} / F\right)$ is a pro-p torsion-free $p$-adic Lie group. Then $X\left(A / F_{\infty}\right)$ is a torsion $\mathcal{O} \llbracket G \rrbracket$-module if and only if $X\left(A^{*} / F_{\infty}\right)$ is a torsion $\mathcal{O} \llbracket G \rrbracket$-module.

The remainder of the subsection will be devoted to the proof of Theorem 4.1.1. By Proposition 2.4.7, we are reduced to proving the following proposition.

Proposition 4.1.3. For every $n \geq 1$, we have

$$
\mu_{\mathcal{O} \llbracket G \rrbracket}\left(X\left(A / F_{\infty}\right) / \pi^{n}\right)=\mu_{\mathcal{O} \llbracket G \rrbracket}\left(X\left(A^{*} / F_{\infty}\right) / \pi^{n}\right) .
$$


Proof. Let $n$ be an arbitrary fixed positive integer. Then for every $m \geq 1$, we have

$$
\begin{aligned}
&\left|\left[G: G_{0}\right]\left(\mu_{\mathcal{O} \llbracket G \rrbracket}\left(X\left(A / F_{\infty}\right) / \pi^{n}\right)-\mu_{\mathcal{O} \llbracket G \rrbracket}\left(X\left(A^{*} / F_{\infty}\right) / \pi^{n}\right)\right) p^{r m}\right| \leq \\
&\left|\left[G: G_{0}\right] \mu_{\mathcal{O} \llbracket G \rrbracket}\left(X\left(A / F_{\infty}\right) / \pi^{n}\right) p^{r m}-\operatorname{ord}_{q}\left(S\left(A / F_{\infty}\right)\left[\pi^{n}\right] G_{m}\right)\right| \\
&++\left[G: G_{0}\right] \mu_{\mathcal{O} \llbracket G \rrbracket}\left(X\left(A^{*} / F_{\infty}\right) / \pi^{n}\right) p^{r m}-\operatorname{ord}_{q}\left(S\left(A^{*} / F_{\infty}\right)\left[\pi^{n}\right]^{G_{m}}\right) \mid \\
&+ \operatorname{ord}_{q}\left(S\left(A\left[\pi^{n}\right] / F_{m}\right)\right)-\operatorname{ord}_{q}\left(S\left(A / F_{\infty}\right)\left[\pi^{n}\right]^{G_{m}}\right) \mid \\
&+ \operatorname{ord}_{q}\left(S\left(A^{*}\left[\pi^{n}\right] / F_{m}\right)\right)-\operatorname{ord}_{q}\left(S\left(A^{*} / F_{\infty}\right)\left[\pi^{n}\right]^{G_{m}}\right) \mid \\
&+ \operatorname{ord}_{q}\left(S\left(A\left[\pi^{n}\right] / F_{m}\right)\right)-\operatorname{ord}_{q}\left(S\left(A^{*}\left[\pi^{n}\right] / F_{m}\right)\right) \mid .
\end{aligned}
$$

The required equality of the proposition will follow once we can show that each of the five quantities on the right is $O\left(p^{(r-1) m}\right)$. The first two quantities are $O\left(p^{(r-1) m}\right)$ by Theorem 2.5.1, We now proceed to show that the third and fourth quantities are $O\left(p^{(r-1) m}\right)$. To show this, we first need to estimate the order of the kernels and cokernels of the maps

$$
S\left(A\left[\pi^{n}\right] / F_{m}\right) \stackrel{r_{m}}{\longrightarrow} S\left(A / F_{m}\right)\left[\pi^{n}\right] \stackrel{s_{m}}{\longrightarrow}\left(S\left(A / F_{\infty}\right)\left[\pi^{n}\right]\right)^{G_{m}} .
$$

One sees easily that ker $r_{m} \subseteq A\left(F_{m}\right) / \pi^{n}$ and ker $s_{m} \subseteq H^{1}\left(G_{m}, A\left(F_{\infty}\right)\right)\left[\pi^{n}\right]$. It is clear that one has $\operatorname{ord}_{q}\left(\operatorname{ker} r_{m}\right) \leq n r$ for every $m$, and therefore, $\operatorname{ord}_{q}\left(\operatorname{ker} r_{m}\right)=O(1)$. On the other hand, it follows from Lemma 4.0.1 that $\operatorname{ord}_{q}\left(H^{1}\left(G_{m}, A\left(F_{\infty}\right)\right)\left[\pi^{n}\right]\right)=O(1)$ (noting that $h_{1}\left(G_{m}\right)$ is a constant function in $m$ ). Thus, one has $\operatorname{ord}_{q}\left(\operatorname{ker} s_{m}\right)=O(1)$.

To estimate coker $r_{m}$ and coker $s_{m}$, one first observes that $\operatorname{ord}_{q}\left(\operatorname{coker} r_{m}\right) \leq \operatorname{ord}_{q}\left(\operatorname{ker} r_{m}^{\prime}\right)$ and that $\operatorname{ord}_{q}\left(\operatorname{coker} s_{m}\right) \leq \operatorname{ord}_{q}\left(\operatorname{ker} s_{m}^{\prime}\right)+\operatorname{ord}_{q}\left(H^{2}\left(G_{m}, A\left(F_{\infty}\right)\right)\left[\pi^{n}\right]\right)$, where $r_{m}^{\prime}$ and $s_{m}^{\prime}$ are given by

$$
\begin{gathered}
r_{m}^{\prime}=\left(r_{m, v_{m}}^{\prime}\right): \bigoplus_{v_{m} \in S_{F_{m}}} H_{s}^{1}\left(F_{m, v_{m}}, A\left[\pi^{n}\right]\right) \longrightarrow \bigoplus_{v_{m} \in S_{F_{m}}} H_{s}^{1}\left(F_{m, v_{m}}, A\right)\left[\pi^{n}\right] ; \\
s_{m}^{\prime}=\left(s_{m, v_{m}}^{\prime}\right): \bigoplus_{v_{m} \in S_{F_{m}}} H_{s}^{1}\left(F_{m, v_{m}}, A\right)\left[\pi^{n}\right] \longrightarrow\left(\underset{m}{\lim } \bigoplus_{v_{m} \in S_{F_{m}}} H_{s}^{1}\left(F_{m, v_{m}}, A\right)\left[\pi^{n}\right]\right)^{G_{m}} .
\end{gathered}
$$

By Lemma 4.0.1, one has that $\operatorname{ord}_{q}\left(H^{2}\left(G_{m}, A\left(F_{\infty}\right)\right)\left[\pi^{n}\right]\right)=O(1)$ (noting that $h_{2}\left(G_{m}\right)$ is a constant function in $m$; in fact, one has $h_{2}\left(G_{m}\right)=r(r-1) / 2$ by [DS+, Theorem 4.35]). To estimate coker $r_{m}^{\prime}$, we first observe that

$$
\begin{aligned}
\operatorname{ker} r_{m, v_{m}}^{\prime} \subseteq & \begin{cases}\operatorname{ker}\left(H^{1}\left(F_{m, v_{m}}, A / A_{v_{m}}\left[\pi^{n}\right]\right) \longrightarrow H^{1}\left(F_{m, v_{m}}, A / A_{v_{m}}\right)\left[\pi^{n}\right]\right) & \text { if } v_{m} \mid p, \\
\operatorname{ker}\left(H^{1}\left(F_{m, v_{m}}^{u r}, A\left[\pi^{n}\right]\right) \longrightarrow H^{1}\left(F_{m, v_{m}}^{u r}, A\right)\left[\pi^{n}\right]\right) & \text { if } v_{m} \nmid p,\end{cases} \\
& = \begin{cases}A / A_{v_{m}}\left(F_{m, v_{m}}\right)\left[\pi^{n}\right] & \text { if } v_{m} \mid p, \\
A\left(F_{m, v_{m}}^{u r}\right)\left[\pi^{n}\right] & \text { if } v_{m} \nmid p,\end{cases}
\end{aligned}
$$


It is now clear from the above that $\operatorname{ord}_{p}\left(\operatorname{ker} r_{m, v_{m}}^{\prime}\right)$ is bounded independently of $m$ and $v_{m}$ (for a fixed $n$ ). Combining these estimates with the fact that the decomposition group of $v$ in $G$ has dimension $\geq 1$ for every $v \in S$ ( since $F_{\infty}$ contains $F^{\text {cyc }}$ ), one then has the estimate $\operatorname{ord}_{q}\left(\operatorname{ker} r_{m}^{\prime}\right)=O\left(p^{(r-1) m}\right)$.

To estimate coker $s_{m}^{\prime}$, we observe that

$$
\operatorname{ker} s_{m, v_{m}}^{\prime} \subseteq \begin{cases}H^{1}\left(G_{m, v_{m}}, A / A_{v_{m}}\left(F_{m, v_{m}}\right)\right)\left[\pi^{n}\right] & \text { if } v_{m} \mid p, \\ H^{1}\left(\operatorname{Gal}\left(F_{\infty, v_{m}} / F_{m, v_{m}}^{u r}\right), A\left(F_{m, v_{m}}\right)\right)\left[\pi^{n}\right] & \text { if } v_{m} \nmid p .\end{cases}
$$

By appealing to Lemma 4.0.1, one verifies easily that $\operatorname{ker} s_{m, v_{m}}^{\prime}$ is bounded independent of $m$ and $v_{m}$ (for a fixed $n$ ). As before, combining these estimates with the fact that the decomposition group of $v$ in $G$ has dimension $\geq 1$ for every $v \in S$ (since $F_{\infty}$ contains $F^{\text {cyc }}$ ), we obtain $\operatorname{ord}_{q}\left(\operatorname{ker} s_{m}^{\prime}\right)=O\left(p^{(r-1) m}\right)$. In conclusion, we have

$$
\operatorname{ord}_{q}\left(S\left(A\left[\pi^{n}\right] / F_{m}\right)\right)=\operatorname{ord}_{q}\left(S\left(A / F_{\infty}\right)\left[\pi^{n}\right]^{G_{m}}\right)+O\left(p^{(r-1) m}\right)
$$

By a similar argument, one also has

$$
\operatorname{ord}_{q}\left(S\left(A^{*}\left[\pi^{n}\right] / F_{m}\right)\right)=\operatorname{ord}_{q}\left(S\left(A^{*} / F_{\infty}\right)\left[\pi^{n}\right]^{G_{m}}\right)+O\left(p^{(r-1) m}\right) .
$$

Therefore, we have shown that the third and fourth quantities are $O\left(p^{(r-1) m}\right)$. For the estimate of the final quantity, we require the following lemma.

Lemma 4.1.4. For every $n$ and $m$, we have

$$
\begin{aligned}
& \frac{\left|S\left(A\left[\pi^{n}\right] / F_{m}\right)\right|}{\left|H^{0}\left(G_{S}\left(F_{m}\right), A\left[\pi^{n}\right]\right)\right|} \times \prod_{v_{m} \mid p}\left|H^{0}\left(F_{m, v_{m}}, A\left[p^{n}\right] / A_{v_{m}}\left[\pi^{n}\right]\right)\right| \\
& \quad=\frac{\left|S\left(A^{*}\left[\pi^{n}\right] / F_{m}\right)\right|}{\left|H^{0}\left(G_{S}\left(F_{m}\right), A^{*}\left[\pi^{n}\right]\right)\right|} \times \prod_{v_{m} \mid p}\left|H^{0}\left(F_{m, v_{m}}, A^{*}\left[\pi^{n}\right] / A_{v_{m}}^{*}\left[\pi^{n}\right]\right)\right|,
\end{aligned}
$$

where the product is taken over all the primes of $F_{m}$ above $p$.

Proof. (Sketch of the proof) This is proven in the same way as [Gr1, Formula (53)] and we give the general idea behind the calculations, leaving the details to the readers. By appealing to the fact that our datum satisfies equality (3.0.1), one can verify the following

$\frac{\left|H^{0}\left(G_{S}\left(F_{m}\right), A\left[\pi^{n}\right]\right)\right|\left|H^{2}\left(G_{S}\left(F_{m}\right), A\left[\pi^{n}\right]\right)\right|}{\left|H^{1}\left(G_{S}\left(F_{m}\right), A\left[\pi^{n}\right]\right)\right|}=\prod_{v_{m} \mid p} \frac{\left|H^{0}\left(F_{m, v_{m}}, A / A_{v_{m}}\left[\pi^{n}\right]\right)\right|\left|H^{2}\left(F_{m, v_{m}}, A / A_{v_{m}}\left[\pi^{n}\right]\right)\right|}{\left|H^{1}\left(F_{m, v_{m}} v, A / A_{v_{m}}\left[\pi^{n}\right]\right)\right|}$

by a global-local Euler characteristic argument. The required equality of the lemma will follow by combining the above with a Poitou-Tate duality argument. 
We continue the proof of our main theorem.

Proof of Theorem 4.1.1 (cont'd). Clearly, the quantities $\left|H^{0}\left(G_{S}\left(F_{m}\right), A\left[p^{n}\right]\right)\right|,\left|H^{0}\left(G_{S}\left(F_{m}\right), A^{*}\left[p^{n}\right]\right)\right|$, $\left|H^{0}\left(F_{m, v_{m}}, A\left[\pi^{n}\right] / A_{v}\left[\pi^{n}\right]\right)\right|$ and $\left|H^{0}\left(F_{m, v_{m}}, A^{*}\left[\pi^{n}\right] / A_{v}^{*}\left[\pi^{n}\right]\right)\right|$ are bounded independently of $m$ and $v_{m}$ (for a fixed $n$ ). Since there are only finite number of primes of $F^{\text {cyc }}$ above $p$, the decomposition group of $v$ in $G$ has at least dimension 1 . Therefore, it follows that $\prod_{v_{m} \mid p}\left|H^{0}\left(F_{m, v_{m}}, A\left[\pi^{n}\right] / A_{v}\left[\pi^{n}\right]\right)\right|$ and $\prod_{v_{m} \mid p}\left|H^{0}\left(F_{m, v_{m}}, A^{*}\left[\pi^{n}\right] / A_{v}^{*}\left[\pi^{n}\right]\right)\right|$ are both $q^{O\left(p^{(r-1) m}\right)}$. Therefore, in conclusion, we have

$$
\operatorname{ord}_{q}\left(S\left(A\left[\pi^{n}\right] / F_{m}\right)\right)=\operatorname{ord}_{q}\left(S\left(A^{*}\left[\pi^{n}\right] / F_{m}\right)\right)+O\left(p^{(r-1) m}\right)
$$

as required. This completes the proof of the theorem.

Remark 4.1.5. (1) If $F_{\infty}$ is a general $p$-adic Lie extension of $F$ (that does not contain $F^{\text {cyc }}$ ) which has the property such that for each prime $v \in S$, the decomposition group of $\operatorname{Gal}\left(F_{\infty} / F\right)$ at $v$ has dimension $\geq 1$, then the argument of Theorem 4.1 .1 carries over to give the same conclusion.

(2) When $F_{\infty}=F^{\text {cyc }}$, Greenberg claimed that $X^{G r}\left(A / F^{\text {cyc }}\right)$ and $X^{G r}\left(A^{*} / F^{\text {cyc }}\right)$ might be pseudo-isomorphic up to an $\iota$-twist (see [Gr1, P. 130, Equation (66)]) and gave some examples where this pseudo-isomorphism can be shown (see discussion after [Gr1, P. 130, Equation (66)]). In view of Lemma 3.1.2, Theorem 4.1.1 may therefore be viewed as providing a positive answer to the $\pi$-primary part of the assertion of Greenberg. (In fact, our result also establishes higher analog of this.)

\subsection{Congruent Galois representations}

As before, let $F_{\infty}$ be an admissible $p$-adic Lie-extension of $F$ whose Galois group is a pro- $p$ torsion-free $p$-adic Lie group of dimension $r$. We write $G=\operatorname{Gal}\left(F_{\infty} / F\right)$. To state our result, we introduce another datum $\left(B,\left\{B_{v}\right\}_{v \mid p},\left\{B_{v}^{+}\right\}_{v \mid \mathbb{R}}\right)$ which satisfies the conditions (a)-(d) as in Section 3. To compare the Selmer groups, we need to expand the set $S$ of primes to contain the ramified primes of $B$. We introduce the following important congruence condition on $A$ and $B$ which allows us to be able to compare the Selmer groups of $A$ and $B$.

$\left(\mathbf{C o n g}_{\mathbf{n}}\right):$ There is an isomorphism $A\left[\pi^{n}\right] \cong B\left[\pi^{n}\right]$ of $G_{S}(F)$-modules which induces a $\operatorname{Gal}\left(\bar{F}_{v} / F_{v}\right)$ isomorphism $A_{v}\left[\pi^{n}\right] \cong B_{v}\left[\pi^{n}\right]$ for every $v \mid p$.

Clearly, $\left(\mathbf{C o n g}_{\mathbf{n}}\right)$ implies $\left(\mathbf{C o n g}_{\mathbf{i}}\right)$ for $i \leq n$. To simplify notation, we will write $\theta_{G}(A)=$ $\theta_{G}\left(X\left(A / F_{\infty}\right)\right)$ and $\theta_{G}(B)=\theta_{G}\left(X\left(B / F_{\infty}\right)\right)$. The following is the second main theorem of the paper. 
Theorem 4.2.1. Let $F_{\infty}$ be an admissible p-adic Lie extension of $F$ whose Galois group is a pro-p torsion-free $p$-adic Lie group. Suppose that $\left(\mathbf{C o n g}_{\theta_{\mathbf{G}}}(\mathbf{A})+\mathbf{1}\right)$ holds and suppose that $X\left(A / F_{\infty}\right)$ is torsion over $\mathcal{O} \llbracket G \rrbracket$. Then $X\left(B / F_{\infty}\right)$ is torsion over $\mathcal{O} \llbracket G \rrbracket$, and $X\left(A / F_{\infty}\right)(\pi)$ and $X\left(B / F_{\infty}\right)(\pi)$ have the same elementary representations.

Proof. By Proposition 2.4.6, it suffices to show that

$$
\mu_{\mathcal{O} \llbracket G \rrbracket}\left(X\left(A / F_{\infty}\right) / \pi^{n}\right)=\mu_{\mathcal{O} \llbracket G \rrbracket}\left(X\left(B / F_{\infty}\right) / \pi^{n}\right)
$$

for $1 \leq n \leq \theta_{G}(A)+1$. Fix such an arbitrary $n$. Then for $m \geq 1$, we have

$$
\begin{aligned}
& \left|\left[G: G_{0}\right]\left(\mu_{\mathcal{O} \llbracket G \rrbracket}\left(X\left(A / F_{\infty}\right) / \pi^{n}\right)-\mu_{\mathcal{O} \llbracket G \rrbracket}\left(X\left(B / F_{\infty}\right) / \pi^{n}\right)\right) p^{r m}\right| \leq \\
& \left|\left[G: G_{0}\right] \mu_{\mathcal{O} \llbracket G \rrbracket}\left(X\left(A / F_{\infty}\right) / \pi^{n}\right) p^{r m}-\operatorname{ord}_{q}\left(S\left(A / F_{\infty}\right)\left[\pi^{n}\right]^{G_{m}}\right)\right| \\
& +\left|\left[G: G_{0}\right] \mu_{\mathcal{O} \llbracket G \rrbracket}\left(X\left(B / F_{\infty}\right) / \pi^{n}\right) p^{r m}-\operatorname{ord}_{q}\left(S\left(B / F_{\infty}\right)\left[\pi^{n}\right]^{G_{m}}\right)\right| \\
& +\operatorname{ord}_{q}\left(S\left(A\left[\pi^{n}\right] / F_{m}\right)\right)-\operatorname{ord}_{q}\left(S\left(A / F_{\infty}\right)\left[\pi^{n}\right]^{G_{m}}\right) \\
& +\operatorname{ord}_{q}\left(S\left(B\left[\pi^{n}\right] / F_{m}\right)\right)-\operatorname{ord}_{q}\left(S\left(B / F_{\infty}\right)\left[\pi^{n}\right]^{G_{m}}\right) \mid \\
& +\left|\operatorname{ord}_{q}\left(S\left(A\left[\pi^{n}\right] / F_{m}\right)\right)-\operatorname{ord}_{q}\left(S\left(B\left[\pi^{n}\right] / F_{m}\right)\right)\right| \text {. }
\end{aligned}
$$

As seen from the argument in the proof of Theorem 4.1.1, the first four quantities on the right of the inequality are $O\left(p^{(r-1) m}\right)$. It remains to estimate the last quantity. By the discussion before this theorem, we have that $\left(\mathbf{C o n g}_{\mathbf{n}}\right)$ holds for $1 \leq n \leq \theta_{G}(A)+1$. This in turn implies that

$$
S\left(A\left[\pi^{n}\right] / F_{m}\right) \cong S\left(B\left[\pi^{n}\right] / F_{m}\right)
$$

for all $m$. In particular, the final quantity on the right of the inequality is zero. Hence we have that

$$
\left|\left[G: G_{0}\right]\left(\mu_{\mathcal{O} \llbracket G \rrbracket}\left(X\left(A / F_{\infty}\right) / \pi^{n}\right)-\mu_{\mathcal{O} \llbracket G \rrbracket}\left(X\left(B / F_{\infty}\right) / \pi^{n}\right)\right) p^{r m}\right|=O\left(p^{(r-1) m}\right)
$$

which implies that

$$
\mu_{\mathcal{O} \llbracket G \rrbracket}\left(X\left(A / F_{\infty}\right) / \pi^{n}\right)=\mu_{\mathcal{O} \llbracket G \rrbracket}\left(X\left(B / F_{\infty}\right) / \pi^{n}\right)
$$

as required.

Remark 4.2.2. If $F_{\infty}$ is a general $p$-adic Lie extension of $F$ (that does not contain $F^{\mathrm{cyc}}$ ) which has the property such that for each prime $v \in S$, the decomposition group of $\operatorname{Gal}\left(F_{\infty} / F\right)$ at $v$ has dimension $\geq 1$, then the argument of Theorem 4.2.1 carries over to give the same conclusion. 


\section{$5 \quad$ Miscellaneous}

\subsection{Some remarks on the $\mathfrak{M}_{H}(G)$-property}

Let $F_{\infty}$ be an admissible $p$-adic Lie extension. As before, we write $G=\operatorname{Gal}\left(F_{\infty} / F\right), H=$ $\operatorname{Gal}\left(F_{\infty} / F^{\text {cyc }}\right)$ and $\Gamma=\operatorname{Gal}\left(F^{\text {cyc }} / F\right)$. We say that an $\mathcal{O} \llbracket G \rrbracket$-module $M$ satisfies the $\mathfrak{M}_{H}(G)$ property if $M_{f}:=M / M(\pi)$ is finitely generated over $\mathcal{O} \llbracket H \rrbracket$. It has been conjectured for certain Galois representations coming from abelian varieties with good ordinary reduction at $p$ or cuspidal eigenforms with good ordinary reduction at $p$, the dual Selmer group associated to such a Galois representation satisfies the $\mathfrak{M}_{H}(G)$-property (see [CF,+ CS,,$\left.\overline{F K}\right]$ ).

For the remainder of this subsection, we will assume that $G$ is a pro- $p$ group of dimension 2 and has no elements of order $p$. As before, $\left(A,\left\{A_{v}\right\}_{v \mid p},\left\{A_{v}^{+}\right\}_{v \mid \mathbb{R}}\right)$ denotes a set of data as defined in Section 3. In preparation for further discussion, we record the following lemma which has a similar proof to that in [CS, Corollary 3.2].

Lemma 5.1.1. Let $F_{\infty}$ be an $S$-admissible p-adic Lie extension whose Galois group is a pro-p group of dimension 2 and has no elements of order $p$. Suppose that $A\left(F^{\mathrm{cyc}}\right)$ is finite. Then the following statements are equivalent.

(a) $X\left(A / F_{\infty}\right)$ satisfies the $\mathfrak{M}_{H}(G)$-property.

(b) $X\left(A / F^{\mathrm{cyc}}\right)$ is a torsion $\mathcal{O} \llbracket \Gamma \rrbracket$-module, $X\left(A / F_{\infty}\right)$ is a torsion $\mathcal{O} \llbracket G \rrbracket$-module and

$$
\mu_{\mathcal{O} \llbracket G \rrbracket}\left(X\left(A / F_{\infty}\right)\right)=\mu_{\mathcal{O} \llbracket \Gamma \rrbracket}\left(X\left(A / F^{\mathrm{cyc}}\right)\right) .
$$

We should mention that the finiteness condition on $A\left(F^{\mathrm{cyc}}\right)$ has been verified in many cases, and therefore, the discussion in this subsection may apply to these situations. In the case of an abelian variety with good ordinary reduction at $p$, this is verified in $[\mathrm{Im}]$ and for a cuspidal eigenform with good ordinary reduction at $p$, this is done in [Su, Proof of Lemma 2.2]. For a more general result on the finiteness condition for $A$ arising from the Galois representation attached to an étale $i$ th-cohomology group (for $i$ odd) of a smooth proper variety with potentially good reduction, we refer readers to [CSW, $\mathrm{KT}$ ].

We now state the next result which compares the structural properties of $X\left(A / F_{\infty}\right)$ and $X\left(A^{*} / F_{\infty}\right)$, where $X\left(A / F_{\infty}\right)$ is the Selmer group associated to the set of data $\left(A,\left\{A_{v}\right\}_{v \mid p},\left\{A_{v}^{+}\right\}_{v \mid \mathbb{R}}\right)$ and $X\left(A^{*} / F_{\infty}\right)$ is the Selmer group associated to $\left(A^{*},\left\{A_{v}^{*}\right\}_{v \mid p},\left\{\left(A^{*}\right)_{v}^{+}\right\}_{v \mid \mathbb{R}}\right)$.

Proposition 5.1.2. Let $F_{\infty}$ be an admissible p-adic Lie extension of $F$, whose Galois group is a pro-p group of dimension 2 and has no elements of order $p$. Furthermore, suppose that $A\left(F^{\mathrm{cyc}}\right)$ and $A^{*}\left(F^{\mathrm{cyc}}\right)$ are finite. Then $X\left(A / F_{\infty}\right)$ satisfies the $\mathfrak{M}_{H}(G)$-property if and only if $X\left(A^{*} / F_{\infty}\right)$ satisfies the $\mathfrak{M}_{H}(G)$-property. 
Proof. It suffices to show that if $X\left(A / F_{\infty}\right)$ satisfies the $\mathfrak{M}_{H}(G)$-property, then $X\left(A^{*} / F_{\infty}\right)$ also satisfies the $\mathfrak{M}_{H}(G)$-property. Suppose that $X\left(A / F_{\infty}\right)$ satisfies the $\mathfrak{M}_{H}(G)$-property. Then by Lemma 5.1.1, we have that $X\left(A / F^{\text {cyc }}\right)$ is a torsion $\mathcal{O} \llbracket \Gamma \rrbracket$-module, $X\left(A / F_{\infty}\right)$ is a torsion $\mathcal{O} \llbracket G \rrbracket$-module and

$$
\mu_{\mathcal{O} \llbracket G \rrbracket}\left(X\left(A / F_{\infty}\right)\right)=\mu_{\mathcal{O} \llbracket \Gamma \rrbracket}\left(X\left(A / F^{\mathrm{cyc}}\right)\right) .
$$

By virtue of Theorem 4.1.1, we then have that $X\left(A^{*} / F^{\text {cyc }}\right)$ is a torsion $\mathcal{O} \llbracket \Gamma \rrbracket$-module, $X\left(A^{*} / F_{\infty}\right)$ is a torsion $\mathcal{O} \llbracket G \rrbracket$-module and

$$
\mu_{\mathcal{O} \llbracket G \rrbracket}\left(X\left(A^{*} / F_{\infty}\right)\right)=\mu_{\mathcal{O} \llbracket \Gamma \rrbracket}\left(X\left(A^{*} / F^{\mathrm{cyc}}\right)\right) .
$$

By appealing to Lemma 5.1.1 again, this in turn implies that $X\left(A^{*} / F_{\infty}\right)$ satisfies the $\mathfrak{M}_{H}(G)$ property.

We also have a similar result as above for congruent representations. Let $\left(B,\left\{B_{v}\right\}_{v \mid p},\left\{B_{v}^{+}\right\}_{v \mid \mathbb{R}}\right)$ be another set of data defined as in Section 3. The next proposition can be proven similarly by combining Theorem 4.2.1 and Lemma 5.1.1.

Proposition 5.1.3. Let $F_{\infty}$ be an admissible p-adic Lie extension of $F$, whose Galois group is a pro-p group of dimension 2 and has no elements of order $p$. Assume that $A\left(F^{\mathrm{cyc}}\right)$ and $B\left(F^{\mathrm{cyc}}\right)$ are finite. Suppose that $\left(\mathbf{C o n g}_{\theta+\mathbf{1}}\right)$ holds, where $\theta=\max \left\{\theta_{\mathcal{O} \llbracket \Gamma \rrbracket}(A), \theta_{\mathcal{O} \llbracket G \rrbracket}(A)\right\}$. Then if $X\left(A / F_{\infty}\right)$ satisfies the $\mathfrak{M}_{H}(G)$-property, so does $X\left(B / F_{\infty}\right)$.

\subsection{Comparing specializations of a big Galois representation}

We apply the main result in Subsection 4.2 to compare the Selmer groups of specializations of a big Galois representation. As before, let $p$ be a prime. We let $F$ be a number field. If $p=2$, we assume further that $F$ has no real primes. Denote $\mathcal{O}$ to be the ring of integers of some finite extension $K$ of $\mathbb{Q}_{p}$. We write $R=\mathcal{O} \llbracket T \rrbracket$ for the power series ring in one variable. Suppose that we are given the following set of data:

(a) $\mathcal{A}$ is a cofinitely generated cofree $R$-module of $R$-corank $d$ with a continuous, $R$-linear $\operatorname{Gal}(\bar{F} / F)$-action which is unramified outside a finite set of primes of $F$.

(b) For each prime $v$ of $F$ above $p, \mathcal{A}_{v}$ is a $\operatorname{Gal}\left(\bar{F}_{v} / F_{v}\right)$-submodule of $\mathcal{A}$ which is cofree of $R$-corank $d_{v}$.

(c) For each real prime $v$ of $F$, we write $\mathcal{A}_{v}^{+}=\mathcal{A}^{\operatorname{Gal}\left(\bar{F}_{v} / F_{v}\right)}$ which we assume to be cofree of $R$-corank $d_{v}^{+}$. 
(d) The following equality

$$
\sum_{v \mid p}\left(d-d_{v}\right)\left[F_{v}: \mathbb{Q}_{p}\right]=d r_{2}(F)+\sum_{v \text { real }}\left(d-d_{v}^{+}\right)
$$

holds. Here $r_{2}(F)$ denotes the number of complex primes of $F$.

For any prime element $f$ of $\mathcal{O} \llbracket T \rrbracket$ such that $\mathcal{O} \llbracket T \rrbracket / f$ is a maximal order, then we can obtain a data $\left(\mathcal{A}[f],\left\{\mathcal{A}_{v}[f]\right\}_{v \mid p},\left\{\mathcal{A}_{v}^{+}[f]\right\}_{v \mid \mathbb{R}}\right)$ in the sense of Section 3 , The next lemma has a easy proof which is left to reader.

Lemma 5.2.1. Let $f$ and $g$ be prime elements of $\mathcal{O} \llbracket T \rrbracket$ with $\pi^{n} \mid(f-g)$ such that $\mathcal{O} \llbracket T \rrbracket / f$ and $\mathcal{O} \llbracket T \rrbracket / g$ are maximal orders. Then $\mathcal{A}\left[f, \pi^{n}\right]=\mathcal{A}\left[g, \pi^{n}\right]$. One also has similar conclusions for $\mathcal{A}_{v}$ and $\mathcal{A}_{v}^{+}$.

The next proposition compares the $\pi$-primary submodules of the dual Selmer groups of various specializations of a big Galois representation. For a real number $x$, we denote $\lceil x\rceil$ to be the smallest integer not less than $x$.

Proposition 5.2.2. Let $F_{\infty}$ be an admissible $p$-adic Lie extension of $F$ such that $G=\operatorname{Gal}\left(F_{\infty} / F\right)$ is uniform pro-p group. Let $f$ be a prime element of $\mathcal{O} \llbracket T \rrbracket$ such that $\mathcal{O}^{\prime}:=\mathcal{O} \llbracket T \rrbracket / f$ is a maximal order. Set $A=\mathcal{A}[f]$ and suppose that $X\left(A / F_{\infty}\right)$ is torsion over $\mathcal{O}^{\prime} \llbracket G \rrbracket$. Set

$$
n:=\left\lceil\frac{\theta_{\mathcal{O}^{\prime} \llbracket G \rrbracket}\left(X\left(A / F_{\infty}\right)\right)+1}{e}\right\rceil,
$$

where $e$ is the ramification index of $\mathcal{O}^{\prime} / \mathcal{O}$. Then for every prime element $g$ of $\mathcal{O} \llbracket T \rrbracket$ with $\pi^{n} \mid f-g$ such that $\mathcal{O} \llbracket T \rrbracket / g$ is isomorphic to $\mathcal{O}^{\prime}$, we have that $X\left(\mathcal{A}[g] / F_{\infty}\right)$ is torsion over $\mathcal{O}^{\prime} \llbracket G \rrbracket$, and that $X\left(A / F_{\infty}\right)\left(\pi^{\prime}\right)$ and $X\left(\mathcal{A}[g] / F_{\infty}\right)\left(\pi^{\prime}\right)$ have the same elementary representations.

Note that by Lemma 3.2 .2 , this proposition may be viewed as a refinement of $[\mathrm{B}$, Theorem 1.2(1), Corollary 4.37(1)]). We now give the proof.

Proof of Proposition 5.2.2. Let $g$ be a prime element of $\mathcal{O} \llbracket T \rrbracket$ which satisfies the hypothesis in the proposition. Let $\pi^{\prime}$ be a prime element of $\mathcal{O}^{\prime}$ and write $B=\mathcal{A}[g]$. It follows from Lemma 5.2 .1 that there is an isomorphism of $G_{S}(F)$-modules $A\left[\pi^{\prime e n}\right] \cong B\left[\pi^{\prime e n}\right]$ which induces an isomorphism of $\operatorname{Gal}\left(\bar{F}_{v} / F_{v}\right)$-modules $A_{v}\left[\pi^{\prime e n}\right] \cong B_{v}\left[\pi^{\prime e n}\right]$ for each prime $v$ of $F$ above $p$. By our hypothesis of $n$, we have en $\geq \theta_{\mathcal{O}^{\prime} \llbracket G \rrbracket}(A)+1$. In particular, the congruence hypothesis $\left(\mathbf{C}_{\theta_{\mathcal{O}^{\prime} \llbracket \mathbf{G} \rrbracket}}(\mathbf{A})+\mathbf{1}\right)$ holds for $A$ and $\mathcal{A}[g]$. Hence the conclusion of the proposition is now immediate from Theorem 4.2.1. 
We end the paper with a proposition which is immediate from an application of Proposition 5.1.3. This proposition is a refinement of [SS, Proposition 8.6] and [B, Corollary 4.37] when the admissible $p$-adic Lie extension is of dimension 2 .

Proposition 5.2.3. Let $F_{\infty}$ be an admissible p-adic Lie extension of $F$, whose Galois group is a pro-p group of dimension 2 and has no elements of order $p$. Let $f$ be a prime element of $\mathcal{O} \llbracket T \rrbracket$ such that $\mathcal{O}^{\prime}:=\mathcal{O} \llbracket T \rrbracket / f$ is a maximal order. Set $A=\mathcal{A}[f]$ and suppose that $X\left(A / F_{\infty}\right)$ belongs to $\mathfrak{M}_{H}(G)$. Set

$$
n:=\left\lceil\frac{\theta+1}{e}\right\rceil,
$$

where $\theta=\max \left\{\theta_{\mathcal{O}^{\prime} \llbracket \Gamma \rrbracket}(A), \theta_{\mathcal{O}^{\prime} \llbracket G \rrbracket}(A)\right\}$ and $e$ is the ramification index of $\mathcal{O}^{\prime} / \mathcal{O}$. Then for every prime element $g$ of $\mathcal{O} \llbracket T \rrbracket$ with $\pi^{n} \mid f-g$ such that $\mathcal{O} \llbracket T \rrbracket / g$ is isomorphic to $\mathcal{O}^{\prime}$, we have that $X\left(\mathcal{A}[g] / F_{\infty}\right)$ belongs to $\mathfrak{M}_{H}(G)$.

\section{References}

[AB] K. Ardakov and K. A. Brown, Primeness, semiprimeness and localisation in Iwasawa algebras, Trans. Amer. Math. Soc. 359(4) (2007) 1499-1515.

[BZ] T. Backhausz and G. Zábrádi, Algebraic functional equations and completely faithful Selmer groups, Int. J. Number Theory 11 (2015) 1233-1257.

[BS] R. Barman and A. Saikia, A note on Iwasawa $\mu$-invariants of elliptic curves, Bull Braz Math Soc, New Series 41(3) (2010) 399-407.

[B] P. Barth, Iwasawa theory for one-parameter families of motives, Int. J. Number Theory 9(2) (2013) 257-319.

[Bh] A. Bhave, Comparison of the $\mu$-invariants of an abelian variety and its dual abelian variety, arXiv:1305.3444v1 [math.NT].

[Ch] A. Chandrakant Sharma, Iwasawa invariants for the False-Tate extension and congruences between modular forms, J. Number Theory 129 (2009) 1893-1911.

$\left[\mathrm{CF}^{+}\right]$J. Coates, T. Fukaya, K. Kato, R. Sujatha and O. Venjakob, The $\mathrm{GL}_{2}$ main conjecture for elliptic curves without complex multiplication, Publ. Math. IHES 101 (2005) 163-208.

[CG] J. Coates and R. Greenberg, Kummer theory for abelian varieties over local fields, Invent. Math. 124 (1996) 129-174.

[CS] J. Coates and R. Sujatha, On the $\mathfrak{M}_{H}(G)$-conjecture, in Non-abelian fundamental groups and Iwasawa theory, ed. J. Coates, M. Kim, F. Pop, M. Saidi and P. Schneider, London Math. Soc. Lecture Note Ser. 393, Cambridge Univ. Press, 2012, pp. 132-161.

[CSW] J. Coates, R. Sujatha and J -P Winterberger, On the Euler-Poincaré characteristics of finite dimensional p-adic Galois representations, Publ. Math. IHES 93 (2001) 107-143.

[CM] A. Cucuo and P. Monsky, Class numbers in $\mathbb{Z}_{p}^{d}$-extensions, Math. Ann. 255(2) (1981) $235-258$. 
[DS+] J. Dixon, M. P. F. Du Sautoy, A. Mann and D. Segal, Analytic Pro-p Groups, 2nd edn, Cambridge Stud. Adv. Math. 38, Cambridge Univ. Press, Cambridge, UK, 1999.

[EPW] M. Emerton, R. Pollack and T. Weston, Variation of Iwasawa invariants in Hida families, Invent. Math. 163 (2006) 523-580.

[FK] T. Fukaya and K. Kato, A formulation of conjectures on $p$-adic zeta functions in noncommutative Iwasawa theory, Amer. Math. Soc. Transl. Ser. 2 219, 2006, 1-85.

[GW] K. R. Goodearl and R. B. Warfield, An introduction to non-commutative Noetherian rings, London Math. Soc. Stud. Texts 61, Cambridge University Press, 2004.

[Gr1] R. Greenberg, Iwasawa theory for p-adic representations, in Algebraic Number Theory-in honor of K. Iwasawa, ed. J. Coates, R. Greenberg, B. Mazur and I. Satake, Adv. Std. in Pure Math. 17, 1989, pp. 97-137.

[Gr2] R. Greenberg, Iwasawa theory for p-adic deformations of motives, Proc. Sympos. Pure Math. 55 (Part 2) (1994) 193-223.

[GV] R. Greenberg and V. Vatsal, On the Iwasawa invariants of elliptic curves, Invent. Math. 142 (2000) $17-63$.

[Ha] Y. Hachimori, Iwasawa $\lambda$-invariants and congruence of Galois representations, J. Ramanujan Math. Soc. 26(2) (2011) 203-217.

[Har1] M. Harris, p-adic representations arising from descent on abelian varieties, Comp. Math. 39 (1979) 177245.

[Har2] M. Harris, Correction to $p$-adic representations arising from descent on abelian varieties, Comp. Math. 121 (2000) 105-108

[Ho1] S. Howson, Euler characteristic as invariants of Iwasawa modules, Proc. London Math. Soc. 85(3) (2002) 634-658.

[Ho2] S. Howson, Structure of central torsion Iwasawa modules, Bull. Soc. Math. France 130(4) (2002) 507-535.

[Hs] M.-L. Hsieh, The algebraic functional equation of Selmer groups for CM fields, J. Number Theory 130 (2010), 1914-1924.

[Im] H. Imai, A remark on the rational points of abelian varieties with values in cyclotomic $\mathbb{Z}_{p}$-extensions, Proc. Japan Acad. 51 (1975) 12-16.

[Iw] K. Iwasawa, On Г-extensions of algebraic number fields, Bull. Amer. Math. Soc. 65 (1959) $183-226$.

[JP] S. Jha and A. Pal, Algebraic functional equation for Hida family, Int. J. Number Theory 10(7) (2014) 1649-1674.

[KT] Y. Kubo and Y. Taguchi, A generalization of a theorem of Imai and its applications to Iwasawa theory, Math. Z. 275(3-4) (2013) 1181-1195.

[LLTT] K. F. Lai, I. Longhi, K. -S. Tan and F. Trihan, Pontryagin duality for Iwasawa modules and abelian varieties, arXiv:1406.5815 [math.NT].

[Lam] T. Y. Lam, Lectures on Modules and Rings, Grad. Texts in Math. 189, Springer, 1999.

[Laz] M. Lazard, Groups analytiques p-adiques, Pub. Math. IHES 26 (1965) 389-603.

[Lim] M. F. Lim, Comparing the Selmer group of a $p$-adic representation and the Selmer group of the Tate dual of the representation, unpublished note, available at arXiv:1405.5289 [math.NT]. 
[Mat] K. Matsuno, Finite $\Lambda$-submodules of Selmer groups of abelian varieties over cyclotomic $\mathbb{Z}_{p}$-extensions, $J$. Number Theory 99(2) (2003) 415-443.

[Mon] P. Monsky, Fine estimate for the growth of $e_{n}$ in $\mathbb{Z}_{p}^{d}$-extensions, in Algebraic Number Theory-in honor of K. Iwasawa, ed. J. Coates, R. Greenberg, B. Mazur and I. Satake, Adv. Std. in Pure Math. 17, 1989, pp. 309-330.

[Nek] J. Nekovár̆, Selmer Complexes, Astérisque 310, 2006.

[NSW] J. Neukirch, A. Schmidt and K. Wingberg, Cohomology of Number Fields, 2nd edn, Grundlehren Math. Wiss. 323, Springer 2008.

[Neu] A. Neumann, Completed group algebras without zero divisors, Arch. Math. 51(6) (1988) $496-499$.

[Oc] T. Ochiai, On the two-variable Iwasawa main conjecture, Comp. Math. 142 (2006) 1157-1200.

[Ser] J -P. Serre, Sur la dimension cohomologique des groupes profinis, Topology 3 (1965) 413-420.

[SS] S. Shekhar and R. Sujatha, On the structure of Selmer groups of $\Lambda$-adic deformations over $p$-adic Lie extensions, Doc. Math. 17 (2012) 573-606.

[Su] R. Sujatha, Iwasawa theory and modular forms, Pure Appl. Math. Q. 2(2) (2006) 519-538.

[V1] O. Venjakob, On the structure theory of the Iwasawa algebra of a p-adic Lie group, J. Eur. Math. Soc. 4(3) (2002) 271-311.

[V2] O. Venjakob, A non-commutative Weierstrass preparation theorem and applications to Iwasawa theory, J. reine angew. Math. 559 (2003) 153-191.

[We] T. Weston, Iwasawa invariants of Galois deformations, Manuscripta Math. 118(2) (2005) 161-180.

[Z1] G. Zábrádi, Characteristic elements, pairings and functional equations over the false Tate curve extension, Math. Proc. Camb. Phil. Soc. 144 (2008) 535-574.

[Z2] G. Zábrádi, Pairings and functional equations over the GL 2 -extension, Proc. London Math. Soc. 101(3) (2010) 893-930. 